\title{
Hydrodynamics from charged black branes
}

\author{
Nabamita Banerjee, ${ }^{a}$ Jyotirmoy Bhattacharya, ${ }^{b}$ Sayantani Bhattacharyya, ${ }^{b}$ \\ Suvankar Dutta, ${ }^{a}$ R. Loganayagam ${ }^{b}$ and P. Surówka ${ }^{c, d}$ \\ ${ }^{a}$ Harish-Chandra Research Institute, \\ Chhatnag Road, Jhunsi, Allahabad-211019, India \\ ${ }^{b}$ Dept. of Theoretical Physics, Tata Institute of Fundamental Research, \\ Homi Bhabha Rd, Mumbai 400005, India \\ ${ }^{c}$ Department of Physics, University of Washington, \\ Seattle, WA 98195-1560, U.S.A. \\ ${ }^{d}$ Institute of Physics, Jagiellonian University, \\ Reymonta 4, 30-059 Kraków, Poland \\ E-mail: nabamita@mri.ernet.in, jyotirmoy@theory.tifr.res.in, \\ sayanta@theory.tifr.res.in, suvankar@mri.ernet.in, \\ nayagam@theory.tifr.res.in, surowka@u. washington.edu
}

ABSTRACT: We extend the recent work on fluid-gravity correspondence to charged blackbranes by determining the metric duals to arbitrary charged fluid configuration up to second order in the boundary derivative expansion. We also derive the energy-momentum tensor and the charge current for these configurations up to second order in the boundary derivative expansion. We find a new term in the charge current when there is a bulk Chern-Simons interaction thus resolving an earlier discrepancy between thermodynamics of charged rotating black holes and boundary hydrodynamics. We have also confirmed that all our expressions are covariant under boundary Weyl-transformations as expected.

KEYwOrds: AdS-CFT Correspondence, Black Holes, Holography and quark-gluon plasmas

ArXiv EPRINT: 0809.2596 


\section{Contents}

1 Introduction 1

2 Notations and conventions $\quad 4$

3 First order hydrodynamics $\quad 6$

$\begin{array}{ll}3.1 \text { Scalars of } \mathrm{SO}(3) \text { at first order } & 7\end{array}$

$\begin{array}{ll}3.2 \text { Vectors of } \mathrm{SO}(3) \text { at first order } & 10\end{array}$

$\begin{array}{ll}3.3 \text { Tensors of } \mathrm{SO}(3) \text { at first order } & 12\end{array}$

$\begin{array}{ll}3.4 & \text { The global metric and the gauge field at first order } \\ 3.5 & \text { The }\end{array}$

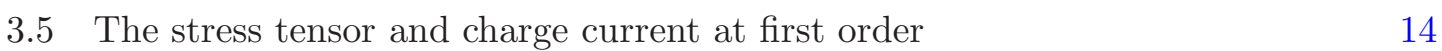

4 Second order hydrodynamics $\quad \mathbf{1 5}$

$\begin{array}{lll}4.1 & \text { Scalars of } \mathrm{SO}(3) \text { at second order } & 17\end{array}$

$\begin{array}{lll}4.2 & \text { Vectors of } \mathrm{SO}(3) \text { at second order } & 18\end{array}$

$\begin{array}{lll}4.3 & \text { Boundary charge current at second order } & 20\end{array}$

4.4 Tensors of $\mathrm{SO}(3)$ at second order 21

$\begin{array}{lll}4.5 & \text { Boundary stress tensor at second order } & 22\end{array}$

5 Discussion $\quad 23$

A Charged conformal fluids and Weyl covariance 24

$\begin{array}{ll}\text { B Source terms in scalar sector: second order } & 27\end{array}$

C Source terms in vector sector: second order $\quad 29$

D Source terms in tensor sector: second order 31

E Comparison with Erdmenger et al. [61] 32

\section{Introduction}

Modern theoretical physics provides mathematically precise descriptions of a bewildering variety of phenomena. The diversity of the phenomena studies by physicists encourages specialization and a consequent divergence in the field. We find it satisfying, however, that this push towards divergence is partially counterbalanced by periodic theoretical discoveries that unify - i.e. discover precise mathematical connections between - distinct fields of physics. In line with this tradition, recent string theory inspired studies of classical gravitational dynamics have found a precise mathematical connection between a long distance 
limit of the Einstein equations of gravity and the Navier Stokes equations of fluid dynamics. More specifically, it has recently been demonstrated that a class of long distance, regular, locally asymptotically $A d S_{d+1}$ solutions to Einstein's equations with a negative cosmological constant is in one to one correspondence with solutions to the charge free Navier Stokes equations in $d$ dimensions $[1-6,8] .^{1}$

The connection between the equations of gravity and fluid dynamics, described above, was demonstrated essentially by use of the method of collective coordinates. The authors of $[1,3-5,8]$ noted that there exists a $d$ parameter set of exact, asymptotically $A d S_{d+1}$ black brane solutions of the gravity equations parameterized by temperature and velocity. They then used the 'Goldstone' philosophy to promote temperatures and velocities to fields. The Navier Stokes equations turn out to be the effective 'chiral Lagrangian equations' of the temperature and velocity collective fields.

This initially surprising connection between gravity in $d+1$ dimensions and fluid dynamics in $d$ dimensions is beautifully explained by the AdS/CFT correspondence. Recall that a particular large $N$ and strong coupling limit of that correspondence relates the dynamics of a classical gravitational theory (a two derivative theory of gravity interacting with other fields) on $\mathrm{AdS}_{d+1}$ space to the dynamics of a strongly coupled conformal field theory in $d$ flat dimensions. Now the dynamics of a conformal field theory, at length scales long compared to an effective mean free path (more accurately an equilibration length scale) is expected to be well described by the Navier Stokes equations. Consequently, the connection between long wavelength solutions of gravity and the equations of fluid dynamics - directly derived in [1] - is a natural prediction of the AdS/CFT correspondence. Using the AdS/CFT correspondence, the stress tensor as a function of velocities and temperatures obtained above from gravity may be interpreted as the fluid stress tensor of the dual boundary field theory in its deconfined phase.

Now consider a conformal field theory that has a conserved charge $Q$ in addition to energy and momentum. This is especially an interesting extension of the hydrodynamics of the uncharged fluids since the hydrodynamics of many real fluids has a global conserved charge which is often just the number of particles that make up the fluid. The long distance dynamics of such a system is expected to be determined by the augmented Navier Stokes equations; $\nabla_{\mu} T^{\mu \nu}=0$ together with $\nabla_{\mu} J_{Q}^{\mu}=0$, where the stress tensor and charge current are now given as functions of the temperature, velocity and charge density, expanded to a given order in the derivative expansion. The bulk dual description of a field theory with a conserved charge always includes a propagating Maxwell field. Consequently the AdS/CFT correspondence suggests asymptotically AdS long wavelength solutions of appropriate modifications of the the Einstein Maxwell equation are in one to one correspondence with solutions of the augmented Navier Stokes equations described above.

This expectation of the previous paragraph also fits well with the collective coordinate intuition described above. Recall that the Einstein Maxwell equations have a well known $d+1$ dimensional set of charged black brane solutions, parameterized by the brane

\footnotetext{
${ }^{1}$ There exists a large literature in deriving linearise hydrodynamics from AdS/CFT. See([9]-[42]). There have been some recent work on hydrodynamics with higher derivative corrections [46, 47].
} 
temperature, charge density and velocity. It seems plausible that the effective Goldstone equations, that arise from the promotion of these $d+1$ dimensional parameters to fields, are simply the augmented Navier Stokes equations. In this paper we verify the expectations via a direct analysis of the relevant bulk equations. More concretely, we generalize the work out in [1] to set up a perturbative scheme to generate long wavelength solutions of the Einstein Maxwell equations plus a Chern Simons term (see below for more details) order by order in the derivative expansion. We also implement this expansion to second order, and thereby find explicit expressions for the stress tensor and charge current of our dual fluid to second order in the derivative expansion.

In this paper we work with the Einstein Maxwell equations augmented by a Chern Simon's term. This is because the equations of IIB SUGRA on $\mathrm{AdS}_{5} \times \mathrm{S}^{5}$ (which is conjectured to be dual to $\mathcal{N}=4$ Yang Mills) with the restriction of equal charges for the three natural Cartans, admit a consistent truncation to this system. Under this truncation, we get the following action

$$
S=\frac{1}{16 \pi G_{5}} \int \sqrt{-g_{5}}\left[R+12-F_{A B} F^{A B}-\frac{4 \kappa}{3} \epsilon^{L A B C D} A_{L} F_{A B} F_{C D}\right]
$$

In the above action the size of the $S_{5}$ has been set to 1 . The value of the parameter $\kappa$ for $\mathcal{N}=4$ Yang Mills is given by $\kappa=1 /(2 \sqrt{3})$ - however, with a view to other potential applications we leave $\kappa$ as a free parameter in all the calculations below. Note in particular that our bulk Lagrangian reduces to the true Einstein Maxwell system at $\kappa=0$.

Our expressions for the charge current and the stress tensor of the fluid are complicated, and are listed in detail in subsequent sections. We would however like to point out an important qualitative feature of our result. Already at first order, and at nonzero $\kappa$, the charge current includes a term proportional to $l^{\alpha} \equiv \epsilon^{\mu \nu \lambda \alpha} u_{\mu} \nabla_{\nu} u_{\lambda}$. The presence of this term in the current resolves an apparent mismatch between the predictions of fluid dynamics and the explicit form of charged rotating black holes in IIB supergravity reported in [45]. Note that due to the presence of the $\epsilon$ symbol, this term is parity odd. However, when accompanied by a flip in the R-charge of the brane, its sign remains unchanged. Consequently, this term is $\mathrm{CP}$ symmetric in agreement with the expectations of CP symmetry of $\mathcal{N}=4$ Yang Mills theory.

As we have explained above, the reduction of boundary field theory dynamics is expected to reduce to field theory dynamics only at long wavelength compared to an effective mean free path or equilibration length scale. All the gravitational constructions of this paper also work only in the same limit. It is consequently of interest to know the functional form of the equilibration length scale of our conformal fluid as a function of intensive fluid parameters.

In the case of $\mathcal{N}=4$ Yang Mills, it follows from 't Hooft scaling and dimensional analysis that, at large $\lambda$, the effective equilibration length scale is given by $l_{m f p}=f(\nu) / T$ where $\nu$ is the dimensionless chemical potential conjugate to the conserved charge of the theory and $T$ being the associated temperature. Explicit computation within gravity demonstrates that $f(\nu)$ is of unit order for generic values of $\nu$. Consequently, at generic values of $\nu$, all the considerations of this paper apply only when all fields vary at distances and times that 
are large compared to the local effective temperature. However, as was explained in detail in [45], the charged fluid we study in this paper has an upper bound on $\nu$ at $\nu_{c}$. At this special value of $\nu$, the black brane becomes extremal. $f(\nu)$ appears to have a simple zero about $\nu_{c}$. Consequently it appears to be possible to scale $\nu$ to $\nu_{c}$ and $T$ to zero simultaneously while keeping $l_{m f p}$ (and all other thermodynamic densities) finite. Thus it would naively appear that the long distance field theory dynamics should be well described by fluid dynamics in this coordinated extremal limit. It turns out, however, that the bulk gravitational solutions described in this paper turn singular in the same limit. We are not completely sure how to interpret this fact. It would certainly be interesting to investigate this further.

Note added. While this draft was in preparation, we became aware of a similar work by Erdmenger et.al. [61].

\section{Notations and conventions}

In this section. we will establish the basic conventions and notations that we will use in the rest of the paper. We start with the five-dimensional action ${ }^{2}$

$$
S=\frac{1}{16 \pi G_{5}} \int \sqrt{-g_{5}}\left[R+12-F_{A B} F^{A B}-\frac{4 \kappa}{3} \epsilon^{L A B C D} A_{L} F_{A B} F_{C D}\right]
$$

which is a consistent truncation of IIB SUGRA Lagrangian on $\mathrm{AdS}_{5} \times \mathrm{S}^{5}$ background with a cosmological constant $\Lambda=-6$ and the Chern-Simons parameter $\kappa=1 /(2 \sqrt{3})$ (See for example, [48-59]). However, for the sake of generality (and to keep track of the effects of the Chern-Simons term), we will work with an arbitrary value of $\kappa$ in the following. In particular, $\kappa=0$ corresponds to a pure Maxwell theory with no Chern-Simons type interactions.

The field equations corresponding to the above action are

$$
\begin{aligned}
G_{A B}-6 g_{A B}+2 & {\left[F_{A C} F^{C}{ }_{B}+\frac{1}{4} g_{A B} F_{C D} F^{C D}\right]=0 } \\
\nabla_{B} F^{A B}+\kappa \epsilon^{A B C D E} F_{B C} F_{D E} & =0
\end{aligned}
$$

where $g_{A B}$ is the five-dimensional metric, $G_{A B}$ is the five dimensional Einstein tensor. These equations admit an AdS-Reisner-Nordström black-brane solution

$$
\begin{aligned}
d s^{2} & =-2 u_{\mu} d x^{\mu} d r-r^{2} V(r, m, q) u_{\mu} u_{\nu} d x^{\mu} d x^{\nu}+r^{2} P_{\mu \nu} d x^{\mu} d x^{\nu} \\
A & =\frac{\sqrt{3} q}{2 r^{2}} u_{\mu} d x^{\mu}
\end{aligned}
$$

where

$$
\begin{aligned}
u_{\mu} d x^{\mu} & =-d v ; \quad V(r, m, q) \equiv 1-\frac{m}{r^{4}}+\frac{q^{2}}{r^{6}} \\
P_{\mu \nu} & \equiv \eta_{\mu \nu}+u_{\mu} u_{\nu}
\end{aligned}
$$

\footnotetext{
${ }^{2}$ We use Latin letters $A, B \in\{r, v, x, y, z\}$ to denote the bulk indices and $\mu, \nu \in\{v, x, y, z\}$ to denote the boundary indices.
} 
with $\eta_{\mu \nu}=\operatorname{diag}(-+++)$ being the Minkowski-metric. Following the procedure elucidated in [1], we shall take this flat black-brane metric as our zeroth order metric/gauge field ansatz and promote the parameters $u_{\mu}, m$ and $q$ to slowly varying fields. ${ }^{3}$

In the course of our calculations, we will often find it convenient to use the following 'rescaled' variables

$$
\rho \equiv \frac{r}{R} ; \quad M \equiv \frac{m}{R^{4}} ; \quad Q \equiv \frac{q}{R^{3}} ; \quad Q^{2}=M-1
$$

where $R$ is the radius of the outer horizon,i.e., the largest positive root of the equation $V=0$. The Hawking temperature, chemical potential and the charge density of this black-brane are given by ${ }^{4}$

$$
T \equiv \frac{R}{2 \pi}\left(2-Q^{2}\right), \quad \mu \equiv \frac{2 \sqrt{3} q}{R^{2}}=2 \sqrt{3} Q R \quad \text { and } \quad n \equiv \frac{\sqrt{3} q}{16 \pi G_{5}} .
$$

In terms of the rescaled variables, the outer and the inner horizon are given by

$$
\rho_{+} \equiv 1 \quad \text { and } \quad \rho_{-} \equiv\left[\left(Q^{2}+1 / 4\right)^{1 / 2}-1 / 2\right]^{1 / 2}
$$

and the extremality condition $\rho_{+}=\rho_{-}$corresponds to $\left(Q^{2}=2, M=3\right)$. We shall assume the black-branes and the corresponding fluids to be non-extremal unless otherwise specified - this corresponds to the regime $0<Q^{2}<2$ or $0<M<3$ which we will assume henceforth.

Using the flat black-brane solutions with slowly varying velocity, temperature and charge fields, our intention is to systematically determine the corrections to the metric and the gauge field in a derivative expansion. More precisely, we expand the metric and the gauge field in terms of derivatives of velocity, temperature and charge fields of the fluid as

$$
\begin{aligned}
& g_{A B}=g_{A B}^{(0)}+g_{A B}^{(1)}+g_{A B}^{(2)}+\ldots \\
& A_{M}=A_{M}^{(0)}+A_{M}^{(1)}+A_{M}^{(2)}+\ldots
\end{aligned}
$$

\footnotetext{
${ }^{3}$ Note that the charge we consider here refers to the Maxwell charge $\int_{\partial S} F_{A B} r^{A} t^{B}$ in the bulk (where $r^{A}$ and $t^{A}$ are respectively the unit radial normal and future pointing time-like normal to the spatial bounday $\partial S$ ). In the presence of a Chern-Simons term in the bulk lagrangian (or alternatively, when the boundary global charge is anomalous), there are other notions of charge (like Page charge - see, for example [60] ) which are employed in the literature. The Page charge in the bulk would be $\int_{\partial S}\left(F_{A B}+2 \kappa \epsilon_{A B}{ }^{C D E} A_{C} F_{D E}\right) r^{A} t^{B}$ in our notation. These other notions of charge in the bulk mirrors the various possible notions of a global charge when it is anomalous in the boundary theory. However, in the rest of the paper, we shall not concern ourselves with these subtleties for the following reason - for the solutions in this paper, $\mathrm{F}$ and hence $A \wedge F$ vanishes when restricted to boundary of AdS. In such a case, the boundary anomaly is turned off and the definition of conserved charge in the boundary is unambiguous (Maxwell charge and Page charge become equal for this subset of solutions). In fact, for a specific value of $\kappa$, this conserved charge refers to the unique R-charge of the boundary super conformal field theory.

${ }^{4} \mathrm{In}$ much of the literature the chemical potential $\mu$ is taken to be the potential difference between the boundary and the horizon. However we have chosen a different normalization for $\mu$ (and hence the charge density $n$ ). we shall elaborate on this point in subsection 3.5.
} 
where $g_{A B}^{(k)}$ and $A_{M}^{(k)}$ contain the k-th derivatives of the velocity, temperature and the charge fields with

$$
\begin{aligned}
g_{A B}^{(0)} d x^{A} d x^{B} & =-2 u_{\mu}(x) d x^{\mu} d r-r^{2} V(r, m(x), q(x)) u_{\mu}(x) u_{\nu}(x) d x^{\mu} d x^{\nu}+r^{2} P_{\mu \nu}(x) d x^{\mu} d x^{\nu} \\
A_{M}^{(0)} d x^{M} & =\frac{\sqrt{3} q(x)}{2 r^{2}} u_{\mu}(x) d x^{\mu} .
\end{aligned}
$$

In order to solve the Einstein-Maxwell-Chern-Simons system of equations, it is necessary to work in a particular gauge for the metric and the gauge fields. Following [1], we choose our gauge to be

$$
g_{r r}=0 ; \quad g_{r \mu} \propto u_{\mu} ; \quad A_{r}=0 ; \quad \operatorname{Tr}\left[\left(g^{(0)}\right)^{-1} g^{(k)}\right]=0 .
$$

Further, in order to relate the bulk dynamics to boundary hydrodynamics, it is useful to parameterise the fluid dynamics in the boundary in terms of a 'fluid velocity' $u_{\mu}$. In case of relativistic fluids with conserved charges, there are two widely used conventions of how the fluid velocity should be defined. In this paper, we will work with the Landau frame velocity where the fluid velocity is defined with reference to the energy transport. In a more practical sense working in the Landau frame amounts to taking the unit time-like eigenvector of the energy-momentum tensor at a point to be the fluid velocity at that point.

Alternatively, one could work in the 'Eckart frame' where the fluid velocity is defined with reference to the charge transport where the unit time-like vector along the charged current to be the definition of fluid velocity. Though the later is often the more natural convention in the context of charged fluids, we choose to use the Landau's convention for the ease of comparison with the other literature. We will leave the conversion to the more natural Eckart frame to future work.

In the next two sections, we will report in some detail the calculations leading to the determination of the metric and the gauge field up to second order in the derivative expansion. This will enable us to determine the boundary stress tensor and charge current up to the second order.

\section{First order hydrodynamics}

In this section, we present the computation of the metric and the gauge field up to first order in derivative expansion, the derivative being taken with respect to the boundary coordinates. We choose the boundary coordinates such that $u^{\mu}=(1,0,0,0)$ at $x^{\mu}$. Since our procedure is ultra local therefore we intend to solve for the metric and the gauge field at first order about this special point $x^{\mu}$. We shall then write the result thus obtained in a covariant form which will be valid for arbitrary choice of boundary coordinates.

In order to implement this procedure we require the zeroth order metric and gauge field expanded up to first order. For this we recall that the parameters $\mathrm{m}, \mathrm{q}$ and the velocities $\left(\beta_{i}\right)$ are functions of the boundary coordinates and therefore admit an expansion in terms 
of the boundary derivatives. These parameters expanded up to first order is given by

$$
\begin{aligned}
m & =m_{0}+x^{\mu} \partial_{\mu} m^{(0)}+\ldots \\
q & =q_{0}+x^{\mu} \partial_{\mu} q^{(0)}+\ldots \\
\beta_{i} & =x^{\mu} \partial_{\mu} \beta_{i}^{(0)}+\ldots
\end{aligned}
$$

Here $m^{(i)}, q^{(i)}, \beta^{(i)}$ refers to the i-th order correction to mass, charge and velocities respectively.

The zeroth order metric expanded about $x^{\mu}$ up to first order in boundary coordinates is given by

$$
\begin{aligned}
d s^{(0)^{2}}= & 2 d v d r-r^{2} V^{(0)}(r) d v^{2}+r^{2} d x_{i} d x^{i} \\
& -2 x^{\mu} \partial_{\mu} \beta_{i}^{(0)} d x^{i} d r-2 x^{\mu} \partial_{\mu} \beta_{i}^{(0)} r^{2}\left(1-V^{(0)}(r)\right) d x^{i} d v \\
& -\left(\frac{-x^{\mu} \partial_{\mu} m^{(0)}}{r^{2}}+\frac{2 q_{0} x^{\mu} \partial_{\mu} q^{(0)}}{r^{4}}\right) d v^{2},
\end{aligned}
$$

where $m_{0}$ and $q_{0}$ are related to the mass and charge of the background blackbrane respectively and

$$
V^{(0)}=1-\frac{m_{0}}{r^{4}}+\frac{q_{0}^{2}}{r^{6}}
$$

Similarly the zeroth order gauge fields expanded about $x^{\mu}$ up to first order is given by

$$
A=-\frac{\sqrt{3}}{2}\left[\left(\frac{q_{0}+x^{\mu} \partial_{\mu} q^{(0)}}{r^{2}}\right) d v-\frac{q_{0}}{r^{2}} x^{\mu} \partial_{\mu} \beta_{i}^{(0)} d x^{i}\right]
$$

Since the background black brane metric preserves an $\mathrm{SO}(3)$ symmetry, ${ }^{5}$ the EinsteinMaxwell equations separate into equations in scalar, transverse vector and the symmetric traceless transverse tensor sectors. This in turn allows us to solve separately for $\mathrm{SO}(3)$ scalar, vector and symmetric traceless tensor components of the metric and the gauge field.

\subsection{Scalars of $\mathrm{SO}(3)$ at first order}

The scalar components of first order metric and gauge field perturbations $\left(g^{(1)}\right.$ and $A^{(1)}$ respectively) are parameterized by the functions $h_{1}(r), k_{1}(r)$ and $w_{1}(r)$ as follows ${ }^{6}$

$$
\begin{aligned}
\sum_{i} g_{i i}^{(1)}(r) & =3 r^{2} h_{1}(r), \\
g_{v v}^{(1)}(r) & =\frac{k_{1}(r)}{r^{2}} \\
g_{v r}^{(1)}(r) & =-\frac{3}{2} h_{1}(r) \\
A_{v}^{(1)}(r) & =-\frac{\sqrt{3} w_{1}(r)}{2 r^{2}}
\end{aligned}
$$

Note that $g_{i i}^{(1)}(r)$ and $g_{v r}^{(1)}(r)$ are related to each other by the gauge choice $\operatorname{Tr}\left[\left(g^{(0)}\right)^{-1} g^{(1)}\right]=0$.

\footnotetext{
${ }^{5}$ Here we are referring to the $\mathrm{SO}(3)$ rotational symmetry in the boundary spatial coordinates.

${ }^{6}$ Here $i$ runs over the boundary spatial coordinates, $\mathrm{v}$ is the boundary time coordinate and $\mathrm{r}$ is the radial coordinate in the bulk.
} 
Constraint equations. We begin by finding the constraint equations that constrain various derivatives velocity,temperature and charge that appear in the first order scalar sector.The constraint equations are obtained by taking a dot of the Einstein and Maxwell equations with the vector dual to the one form $d r$. If we denote the Einstein and the Maxwell equations by $E_{A B}=0$ and $M_{A B}=0$, then there are three constraint relations.

Two of them come from Einstein equations. They are given by

$$
g^{r r} E_{v r}+g^{r v} E_{v v}=0
$$

and

$$
g^{r r} E_{r r}+g^{r v} E_{v r}=0
$$

and the third constraint relation comes from Maxwell equations and is given by

$$
g^{r r} M_{r}+g^{r v} M_{v}=0
$$

Equation (3.5) reduces to

$$
\partial_{v} m^{(0)}=-\frac{4}{3} m_{0} \partial_{i} \beta_{i}^{(0)}
$$

which is same as the conservation of energy in the boundary at the first order in the derivative expansion, i.e., the above equation is identical to the constraint (scalar component of the constraint in this case)

$$
\partial_{\mu} T_{(0)}^{\mu \nu}=0
$$

on the allowed boundary data.

The second constraint equation (3.6) in scalar sector implies a relation between $h_{1}(r)$ and $k_{1}(r)$.

$$
2 \partial_{i} \beta_{i}^{(0)} r^{5}+12 r^{6} h_{1}(r)+4 q_{0} w_{1}(r)-m_{0} r^{3} h_{1}^{\prime}(r)+3 r^{7} h_{1}^{\prime}(r)-r^{3} k_{1}^{\prime}(r)-2 q_{0} r w_{1}^{\prime}(r)=0 .
$$

The constraint relation coming from Maxwell equation (See eq. (3.7)) gives

$$
\partial_{v} q^{(0)}=-q_{0} \partial_{i} \beta_{i}^{(0)}
$$

This equation can be interpreted as the conservation of boundary current density at the first order in the derivative expansion.

$$
\partial_{\mu} J_{(0)}^{\mu}=0 .
$$

We now proceed to find the scalar part of the metric dual to a fluid configuration which obeys the above constraints. 
Dynamical equations and their solutions. Among the Einstein equations four are $\mathrm{SO}(3)$ scalars (namely the $v v, r v, r r$ components and the trace over the boundary spatial part). Further the $r$ and $v$-components of the Maxwell equations constitute two other equations in this sector. Two specific linear combination of the $r r$ and $v v$ components of the Einstein equations constitute the two constraint equations in (3.8). Further, a linear combination of the $r$ and $v$-components of the Maxwell equations appear as a constraint equation in (3.11). Now among the six equations in the scalar sector we can use any three to solve for the unknown functions $h_{1}(r), k_{1}(r)$ and $w_{1}(r)$ and we must make sure that the solution satisfies the rest. The simplest two equations among these dynamical equations are

$$
5 h_{1}^{\prime}(r)+r h_{1}^{\prime \prime}(r)=0
$$

which comes from the $r r$-component of the Einstein equation and

$$
6 q_{0} h_{1}^{\prime}(r)+w_{1}^{\prime}(r)-r w_{1}^{\prime \prime}(r)=0
$$

which comes from the $r$-components of the Maxwell equation. We intend to use these dynamical equations (3.13), (3.14) along with one of the constraint equations in (3.8) to solve for the unknown functions $h_{1}(r), k_{1}(r)$ and $w_{1}(r)$.

Solving (3.13) we get

$$
h_{1}(r)=\frac{C_{h_{1}}^{1}}{r^{4}}+C_{h_{1}}^{2}
$$

where $C_{h_{1}}^{1}$ and $C_{h_{1}}^{2}$ are constants to be determined. We can set $C_{h_{1}}^{2}$ to zero as it will lead to a non-normalizable mode of the metric. We then substitute the solution for $h_{1}(r)$ from (3.15) into (3.14) and solve the resultant equation for $w_{1}(r)$. The solution that we obtain is given by

$$
w_{1}(r)=C_{w_{1}}^{1} r^{2}+C_{w_{1}}^{2}-q_{0} \frac{C_{h_{1}}^{1}}{r^{4}}
$$

Here again $C_{w_{1}}^{1}, C_{w_{2}}^{2}$ are constants to be determined. Again $C_{w_{1}}^{1}$ corresponds to a nonnormalizable mode of the gauge field and therefore can be set to zero.

Finally plugging in these solutions for $h_{1}(r)$ and $w_{1}(r)$ into one of the constraint equations in (3.8) and then solving the subsequent equation we obtain

$$
k_{1}(r)=\frac{2}{3} r^{3} \partial_{i} \beta_{i}^{(0)}+C_{k_{1}}-\frac{2 q 0}{r^{2}} C_{w_{1}}^{2}+\left(\frac{2 q_{0}^{2}}{r^{6}}-\frac{m_{0}}{r^{4}}\right) C_{h_{1}}^{1}
$$

Now the constants $C_{k_{1}}$ and $C_{w_{1}}^{2}$ may be absorbed into redefinitions of mass $\left(m_{0}\right)$ and charge $\left(q_{0}\right)$ respectively and hence may be set to zero. Further we can gauge away the constant $C_{h_{1}}^{1}$ by the following redefinition of the $\mathrm{r}$ coordinate

$$
r \rightarrow r\left(1+\frac{C}{r^{4}}\right)
$$

$C$ being a suitably chosen constant. 
Thus we conclude that all the arbitrary constants in this sector can be set to zero and therefore our solutions may be summarized as

$$
h_{1}(r)=0, \quad w_{1}(r)=0, \quad k_{1}(r)=\frac{2}{3} r^{3} \partial_{i} \beta_{i}^{(0)} .
$$

In terms of the first order metric and gauge field this result reduces to

$$
\begin{aligned}
\sum_{i} g_{i i}^{(1)}(r) & =0, \\
g_{v v}^{(1)}(r) & =\frac{2}{3} r \partial_{i} \beta_{i}^{(0)}, \\
g_{v r}^{(1)}(r) & =0, \\
A_{v}^{(1)}(r) & =0 .
\end{aligned}
$$

Now, we proceed to solving the equations in the vector sector.

\subsection{Vectors of $\mathrm{SO}(3)$ at first order}

The vector components of metric and gauge field $g^{(1)}$ and $A^{(1)}$ are parameterized by the functions $j_{i}^{(1)}(r)$ and $g_{i}^{(1)}(r)$ as follows

$$
\begin{aligned}
& g_{v i}^{(1)}(r)=\left(\frac{m_{0}}{r^{2}}-\frac{q_{0}^{2}}{r^{4}}\right) j_{i}^{(1)}(r) \\
& A_{i}^{(1)}(r)=-\left(\frac{\sqrt{3} q_{0}}{2 r^{2}}\right) j_{i}^{(1)}(r)+g_{i}^{(1)}(r)
\end{aligned}
$$

Now we intend to solve for the functions $j_{i}^{(1)}(r)$ and $g_{i}^{(1)}(r)$.

Constraint equations. The constraint equations in the vector sector comes only from the Einstein equation. So there is only one constraint equation in this sector. It is given by

$$
g^{r r} E_{r i}+g^{r v} E_{v i}=0
$$

which implies

$$
\partial_{i} m^{(0)}=-4 m_{0} \partial_{v} \beta_{i}^{(0)} .
$$

These equations also follow from the conservation of boundary stress tensor at first order. We shall use this constraint equation to simplify the dynamical equations in the vector sector.

Dynamical equations and their solutions. In the vector sector we have two equations from Einstein equations (the $r i$ and vi-components) and one from Maxwell equations (the $i$ th-component). ${ }^{7}$

\footnotetext{
${ }^{7}$ Note that a linear combination of the $r i$ and $v i$-components of the Einstein equation appear as the constraint equation in (3.22).
} 
The dynamical equation obtained from the vi-component of the Einstein equations is given by

$$
\left(q_{0}^{2}-3 m_{0} r^{2}\right) \frac{d j_{i}^{(1)}(r)}{d r}+4 \sqrt{3} q_{0} r^{2} \frac{d g_{i}(1)(r)}{d r}+\left(m_{0} r^{2}-q_{0}^{2}\right) r \frac{d^{2} j_{i}^{(1)}(r)}{d r^{2}}=-3 r^{4} \partial_{v} \beta_{i}^{(0)} .
$$

Also the dynamical equation from the $i$ th-component of the Maxwell equation is given by

$$
\begin{aligned}
& r\left[2\left(r^{6}-m_{0} r^{2}+q_{0}^{2}\right) \frac{d^{2} g_{i}^{(1)}}{d r^{2}} r^{2}+\left(6 r^{7}+2 m_{0} r^{3}-6 q_{0}^{2} r\right) \frac{d g_{i}^{(1)}(r)}{d r}\right] \\
& -\sqrt{3} q_{0} r\left(r^{6}-m_{0} r^{2}+q_{0}^{2}\right) \frac{d^{2} j_{i}^{(1)}(r)}{d r^{2}}+\sqrt{3} q_{0}\left(r^{6}-3 m_{0} r^{2}+5 q_{0}^{2}\right) \frac{d j_{i}^{(1)}(r)}{d r} \\
& \quad=\sqrt{3}\left(q_{0} \partial_{v} \beta_{i}^{(0)}+\partial_{i} q^{(0)}\right) r^{3}-24 q_{0}^{2} \kappa r l_{i}^{(0)}
\end{aligned}
$$

where $l_{i}$ is defined as

$$
l_{i} \equiv \epsilon_{i j k} \partial_{j} \beta_{k} .
$$

Now in order to solve this coupled set of differential equations (3.23) and (3.24) we shall substitute $g_{i}^{(1)}(r)$ obtained from (3.23) into (3.24) and solve the resultant equation for $j_{i}^{(1)}(r)$. For any function $j_{i}^{(1)}(r)$, using $(3.23) g_{i}^{(1)}(r)$ may be expressed as

$$
g_{i}^{(1)}(r)=\left(C_{g}\right)_{i}+\frac{1}{4 \sqrt{3} q_{0}}\left(-\partial_{v} \beta_{i}^{(0)} r^{3}+4 m_{0} j_{i}^{(1)}(r)-\frac{\left(m_{0} r^{2}-q_{0}^{2}\right) \frac{d j_{i}^{(1)}(r)}{d r}}{r}\right)
$$

Here $\left(C_{g}\right)_{i}$ is an arbitrary constant. It corresponds to non normalizable mode of the gauge field and hence may be set to zero.

Substituting this expression for $g_{i}^{(1)}(r)$ into (3.24) we obtain the following differential equation for $j_{i}^{(1)}(r)$

$$
\begin{aligned}
& \left(35 q_{0}^{4}+5 r^{2}\left(r^{4}-6 m_{0}\right) q_{0}^{2}+3 m_{0} r^{4}\left(3 r^{4}+m_{0}\right)\right) \frac{d j_{i}^{(1)}(r)}{d r} \\
& r\left(-11 q_{0}^{4}-\left(5 r^{6}-14 m_{0} r^{2}\right) q_{0}^{2}-m_{0} r^{4}\left(r^{4}+3 m_{0}\right)\right) \frac{d^{2} j_{i}^{(1)}(r)}{d r^{2}} \\
& +r^{2}\left(q_{0}^{2}-m_{0} r^{2}\right)\left(r^{6}-m_{0} r^{2}+q_{0}^{2}\right) \frac{d^{3} j_{i}^{(1)}(r)}{d r^{3}} \\
& \quad=\frac{1}{\sqrt{3}}\left(6 \sqrt{3} q_{0} \partial_{i} q^{(0)} r^{4}+3 \sqrt{3} \partial_{v} \beta_{i}^{(0)}\left(5 r^{6}-m_{0} r^{2}+q_{0}^{2}\right) r^{4}-144 r l_{i}^{(0)} q_{0}^{3} \kappa\right)
\end{aligned}
$$

The solution to this equation is given by,

$$
\begin{aligned}
j_{i}^{(1)}(r)= & \left(C_{j}^{1}\right)_{i}+\frac{\left(C_{j}^{2}\right)_{i} r^{2}}{\frac{m_{0}}{r^{2}}-\frac{q_{0}^{2}}{r^{4}}}+\frac{r \partial_{v} \beta_{i}^{(0)}}{\frac{m_{0}}{r^{2}}-\frac{q_{0}^{2}}{r^{4}}} \\
& +\frac{\sqrt{3} l_{i}^{(0)} q_{0}^{3} \kappa}{m_{0}\left(\frac{m_{0}}{r^{2}}-\frac{q_{0}^{2}}{r^{4}}\right) r^{4}}+\frac{6 r^{2} q_{0}\left(\partial_{i} q^{(0)}+3 q_{0} \partial_{v} \beta_{i}^{(0)}\right)}{R^{7}\left(\frac{m_{0}}{r^{2}}-\frac{q_{0}^{2}}{r^{4}}\right)} F_{1}\left(\frac{r}{R}, \frac{m_{0}}{R^{4}}\right),
\end{aligned}
$$


where again $\left(C_{j}^{1}\right)_{i}$ and $\left(C_{j}^{2}\right)_{i}$ are arbitrary constants. $\left(C_{j}^{2}\right)_{i}$ corresponds to a nonnormalizable mode of the metric and so is set to zero. $\left(C_{j}^{1}\right)_{i}$ can be absorbed into a redefinition of the velocities and hence is also set to zero.

Here the function $F_{1}\left(\frac{r}{R}, \frac{m_{0}}{R^{4}}\right)$ is given by ${ }^{8}$

$$
F_{1}(\rho, M) \equiv \frac{1}{3}\left(1-\frac{M}{\rho^{4}}+\frac{Q^{2}}{\rho^{6}}\right) \int_{\rho}^{\infty} d p \frac{1}{\left(1-\frac{M}{p^{4}}+\frac{Q^{2}}{p^{6}}\right)^{2}}\left(\frac{1}{p^{8}}-\frac{3}{4 p^{7}}\left(1+\frac{1}{M}\right)\right),
$$

where $Q^{2}=M-1$.

Substituting this result for $j_{i}^{(1)}(r)$ into (3.26) we obtain the following expression for $g_{i}^{(1)}(r)$

$$
\begin{aligned}
g_{i}^{(1)}(r)= & \frac{\sqrt{3} r^{3} \sqrt{R^{2}\left(m_{0}-R^{4}\right)}}{2\left(m_{0}(r-R)(r+R)+R^{6}\right)}\left(\partial_{v} \beta_{i}^{(0)}\right)+\frac{3 R^{2} \kappa\left(m_{0}-R^{4}\right)}{2\left(m_{0}\left(r^{2}-R^{2}\right)+R^{6}\right)} l_{i} \\
& -\frac{\sqrt{3} r^{4}\left(r\left(m_{0}\left(r^{2}-R^{2}\right)+R^{6}\right) F_{1}^{(1,0)}\left(\frac{r}{R}, \frac{m_{0}}{R^{4}}\right)+\left(6 R^{7}-6 m_{0} R^{3}\right) F_{1}\left(\frac{r}{R}, \frac{m_{0}}{R^{4}}\right)\right)}{2 R^{8}\left(m_{0}\left(r^{2}-R^{2}\right)+R^{6}\right)} \\
& \times\left(\partial_{i} q^{(0)}+3 q_{0} \partial_{v} \beta_{i}^{(0)}\right)
\end{aligned}
$$

where we use the notation $f^{(i, j)}(\alpha, \beta)$ to denote the partial derivative $\partial^{i+j} f / \partial \alpha^{i} \partial \beta^{j}$ of the function $f$.

Plugging back $j_{i}^{(1)}(r)$ and $g_{i}^{(1)}(r)$ back into (3.20) we conclude that the first order metric and gauge field in the vector sector is given by

$$
\begin{aligned}
& g_{v i}^{(1)}(r)=r \partial_{v} \beta_{i}^{(0)}+\frac{\sqrt{3} l_{i}^{(0)} q_{0}^{3} \kappa}{m_{0} r^{4}}+\frac{6 r^{2}}{R^{7}} q_{0}\left(\partial_{i} q^{(0)}+3 q_{0} \partial_{v} \beta_{i}^{(0)}\right) F_{1}\left(\frac{r}{R}, \frac{m_{0}}{R^{4}}\right) \\
& A_{i}^{(1)}(r)=-\frac{\sqrt{3} r^{5} F_{1}^{(1,0)}\left(\frac{r}{R}, \frac{m_{0}}{R^{4}}\right)}{2 R^{8}}\left(\partial_{i} q^{(0)}+3 q_{0} \partial_{v} \beta_{i}^{(0)}\right)+\frac{3 R \kappa \sqrt{m_{0}-R^{4}} \sqrt{R^{2}\left(m_{0}-R^{4}\right)}}{2 m_{0} r^{2}} l_{i}
\end{aligned}
$$

\subsection{Tensors of $\mathrm{SO}(3)$ at first order}

The tensor components of the first order metric is parameterized by the function $\alpha_{i j}^{(1)}(r)$ such that

$$
g_{i j}^{(1)}=r^{2} \alpha_{i j}^{(1)} .
$$

The gauge field does not have any tensor components therefore in this sector there is only one unknown function to be determined.

There are no constraint equations in this sector and the only dynamical equation is obtained from the $i j$-component of the Einstein equation. This equation is given by

$$
r\left(r^{6}-m_{0} r^{2}+q_{0}^{2}\right) \frac{d^{2} \alpha_{i j}(r)}{d r^{2}}-\left(-5 r^{6}+m_{0} r^{2}+q_{0}^{2}\right) \frac{d \alpha_{i j}(r)}{d r}=-6 \sigma_{i j}^{(0)} r^{4}
$$

where $\sigma_{i j}$ is given by

$$
\sigma_{i j}^{(0)}=\frac{1}{2}\left(\partial_{i} \beta_{j}^{(0)}+\partial_{j} \beta_{i}^{(0)}\right)-\frac{1}{3} \partial_{k} \beta_{k}^{(0)} \delta_{i j}
$$

\footnotetext{
${ }^{8}$ Although the expression for $F_{1}\left(\frac{r}{R}, \frac{m_{0}}{R^{4}}\right)$ is very complicated but it satisfies some identities. One can use those identities to perform practical calculations with this function.
} 
The solution to equation (3.33) obtained by demanding regularity at the future event horizon and appropriate normalizability at infinity. The solution is given by

$$
\alpha_{i j}^{(1)}=\frac{2}{R} \sigma_{i j} F_{2}\left(\frac{r}{R}, \frac{m_{0}}{R^{4}}\right)
$$

where the function $F_{2}(\rho, M)$ is given by

$$
F_{2}(\rho, M) \equiv \int_{\rho}^{\infty} \frac{p\left(p^{2}+p+1\right)}{(p+1)\left(p^{4}+p^{2}-M+1\right)} d p
$$

with $M \equiv m / R^{4}$ as before.

Thus the tensor part of the first order metric is determined to be

$$
g_{i j}^{(1)}=\frac{2 r^{2}}{R} \sigma_{i j} F_{2}\left(\frac{r}{R}, \frac{m_{0}}{R^{4}}\right) .
$$

\subsection{The global metric and the gauge field at first order}

In this subsection, we gather the results of our previous sections to write down the entire metric and the gauge field accurate up to first order in the derivative expansion.

We obtain the metric as

$$
\begin{aligned}
d s^{2}= & g_{A B} d x^{A} d x^{B} \\
= & -2 u_{\mu} d x^{\mu} d r-r^{2} V u_{\mu} u_{\nu} d x^{\mu} d x^{\nu}+r^{2} P_{\mu \nu} d x^{\mu} d x^{\nu} \\
& -2 u_{\mu} d x^{\mu} r\left[u^{\lambda} \partial_{\lambda} u_{\nu}-\frac{\partial_{\lambda} u^{\lambda}}{3} u_{\nu}\right] d x^{\nu}+\frac{2 r^{2}}{R} F_{2}(\rho, M) \sigma_{\mu \nu} d x^{\mu} d x^{\nu} \\
& -2 u_{\mu} d x^{\mu}\left[\frac{\sqrt{3} \kappa q^{3}}{m r^{4}} l_{\nu}+\frac{6 q r^{2}}{R^{7}} P_{\nu}^{\lambda} \mathcal{D}_{\lambda} q F_{1}(\rho, M)\right] d x^{\nu}+\ldots \\
A= & {\left[\frac{\sqrt{3} q}{2 r^{2}} u_{\mu}+\frac{3 \kappa q^{2}}{2 m r^{2}} l_{\mu}-\frac{\sqrt{3} r^{5}}{2 R^{8}} P_{\mu}^{\lambda} \mathcal{D}_{\lambda} q F_{1}^{(1,0)}(\rho, M)\right] d x^{\mu}+\ldots }
\end{aligned}
$$

where $\mathcal{D}_{\lambda}$ is the weyl covarient derivative defined in appendix A. We also have defined

$$
\begin{aligned}
V & \equiv 1-\frac{m}{r^{4}}+\frac{q^{2}}{r^{6}} ; \quad l^{\mu} \equiv \epsilon^{\nu \lambda \sigma \mu} u_{\nu} \partial_{\lambda} u_{\sigma} ; \quad P_{\mu}^{\lambda} \mathcal{D}_{\lambda} q \equiv P_{\mu}^{\lambda} \partial_{\lambda} q+3\left(u^{\lambda} \partial_{\lambda} u_{\mu}\right) q ; \quad \rho \equiv \frac{r}{R} \\
\sigma^{\mu \nu} & \equiv P^{\mu \alpha} P^{\nu \beta} \partial_{(\alpha} u_{\beta)}-\frac{1}{3} P^{\mu \nu} \partial_{\alpha} u_{\alpha} ; \quad M \equiv \frac{m}{R^{4}} ; \quad Q \equiv \frac{q}{R^{3}} ; \quad Q^{2}=M-1
\end{aligned}
$$

and

$$
\begin{aligned}
& F_{1}(\rho, M) \equiv \frac{1}{3}\left(1-\frac{M}{\rho^{4}}+\frac{Q^{2}}{\rho^{6}}\right) \int_{\rho}^{\infty} d p \frac{1}{\left(1-\frac{M}{p^{4}}+\frac{Q^{2}}{p^{6}}\right)^{2}}\left(\frac{1}{p^{8}}-\frac{3}{4 p^{7}}\left(1+\frac{1}{M}\right)\right) \\
& F_{2}(\rho, M) \equiv \int_{\rho}^{\infty} \frac{p\left(p^{2}+p+1\right)}{(p+1)\left(p^{4}+p^{2}-M+1\right)} d p .
\end{aligned}
$$




\subsection{The stress tensor and charge current at first order}

In this section, we obtain the stress tensor and the charge current from the metric and the gauge field. The stress tensor can be obtained from the extrinsic curvature after subtraction of the appropriate counterterms $[43,44]$. We get the first order stress tensor as

$$
T_{\mu \nu}=p\left(\eta_{\mu \nu}+4 u_{\mu} u_{\nu}\right)-2 \eta \sigma_{\mu \nu}+\ldots
$$

where the fluid pressure $p$ and the viscosity $\eta$ are given by the expressions

$$
p \equiv \frac{M R^{4}}{16 \pi G_{5}} ; \quad \eta \equiv \frac{R^{3}}{16 \pi G_{5}}=\frac{s}{4 \pi}
$$

where $s$ is the entropy density of the fluid obtained from the Bekenstein formula.

To obtain the charge current, we use

$$
J_{\mu}=\lim _{r \rightarrow \infty} \frac{r^{2} A_{\mu}}{8 \pi G_{5}}=n u_{\mu}-\mathfrak{D} P_{\mu}^{\nu} \mathcal{D}_{\nu} n+\xi l_{\mu}+\ldots
$$

where the charge density $n$, the diffusion constant $\mathfrak{D}$ and an additional transport coefficient $\xi$ for the fluid under consideration are given by ${ }^{9}$

$$
n \equiv \frac{\sqrt{3} q}{16 \pi G_{5}} ; \quad \mathfrak{D}=\frac{1+M}{4 M R} ; \quad \xi \equiv \frac{3 \kappa q^{2}}{16 \pi G_{5} m}
$$

We note that when the bulk Chern-Simons coupling $\kappa$ is non-zero, apart from the conventional diffusive transport, there is an additional non-dissipative contribution to the charge current which is proportional to the vorticity of the fluid. To the extent we know of, this is a hitherto unknown effect in the hydrodynamics which is exhibited by the conformal fluid made of $\mathcal{N}=4 \mathrm{SYM}$ matter. It would be interesting to find a direct boundary reasoning that would lead to the presence of such a term - however, as of yet, we do not have such an explanation and we hope to return to this issue in future.

The presence of such an effect was indirectly observed by the authors of [45] where they noted a discrepancy between the thermodynamics of charged rotating AdS black holes and the fluid dynamical prediction with the third term in the charge current absent. We have verified that this discrepancy is resolved once we take into account the effect of the third term in the thermodynamics of the rotating $\mathcal{N}=4 \mathrm{SYM}$ fluid. In fact, one could go further and compare the first order metric that we have obtained with rotating black hole metrics written in an appropriate gauge. We have done this comparison up to first order and we find that the metrics agree up to that order.

\footnotetext{
${ }^{9}$ Here we have taken the chemical potential $\mu=2 \sqrt{3} Q R$ which determines the normalization factor of the charge density $n$ (because thermodynamics tells us $n \mu=4 p-T s$ ) which in turn determines the normalization of $J_{\mu}$. Note that due to the difference in $\mu$ with [61], our normalization of $J_{\mu}$ is different from that in [61].
} 


\section{Second order hydrodynamics}

In this section we will find out the metric, stress tensor and charge current at second order in derivative expansion. We will follow the same procedure as in [1] but in presence charge parameter $q$.

The metric and gauge field perturbations at second order that we consider are

$$
g_{\alpha \beta}^{(2)} d x^{\alpha} d x^{\beta}=-3 h_{2}(r) d v d r+r^{2} h_{2}(r) d x^{i} d x_{i}+\frac{k_{2}(r)}{r^{2}} d v^{2}+12 r^{2} j_{i}^{(2)} d v d x^{i}+r^{2} \alpha_{i j}^{(2)} d x^{i} d x^{j}
$$

and

$$
\begin{aligned}
& A_{v}^{(2)}=-\frac{\sqrt{3}}{2 r^{2}} w_{2}(r) \\
& A_{i}^{(2)}=\frac{\sqrt{3}}{2} r^{5} g_{i}^{(2)}(r) d x^{i} .
\end{aligned}
$$

Here we have used a little different parameterizations (from first order) for metric and gauge field perturbations in the vector sector. We found that this aids in writting the corresponding dynamical equations for $j_{i}^{(2)}(r)$ and $g_{i}^{(2)}(r)$ in a more tractable form (as we will see later).

Like neutral black brane case, here also we will list all the source terms (second order in derivative expansion) which will appear on the right hand side of the constraint dynamical equations in scalar, vector and tensor sectors. These source terms are built out of second derivatives of $m, q$ and $\beta$ or square of first derivatives of these three fields. We can group these source terms according to their transformation properties under $\mathrm{SO}(3)$ group. A complete list has been provided in table 1 . In the table the quantities $l_{i}$ and $\sigma_{i j}$ are defined to be

$$
l_{i}=\epsilon_{i j k} \partial_{j} \beta_{k}, \quad \sigma_{i j}=\frac{1}{2}\left(\partial_{i} \beta_{j}+\partial_{j} \beta_{k}\right)-\frac{1}{3} \delta_{i j} \partial_{k} \beta_{k} .
$$

In table 1 we have already employed the first order conservation relations i.e. equation. (3.9) and (3.10). Using these two relations we have eliminated the first derivatives of $m$ and $q$. However at second order in derivative expansion we also have the relations

$$
\partial_{\mu} \partial_{\nu} T_{(0)}^{\mu \nu}=0
$$

and

$$
\partial_{\lambda} \partial_{\mu} J_{(0)}^{\mu}=0
$$

The equations (4.4) and (4.5) imply some relations between the second order source 


\begin{tabular}{|c|c|c|}
\hline 1 of $\mathrm{SO}(3)$ & 3 of $\mathrm{SO}(3)$ & 5 of $\mathrm{SO}(3)$ \\
\hline $\mathrm{S} 1=\partial_{v}^{2} m$ & $\mathrm{~V} 1_{i}=\partial_{i} \partial_{v} m$ & $\mathrm{~T} 1_{i j}=\partial_{i} \partial_{j} m-\frac{1}{3} \mathrm{~s} 3 \delta_{i j}$ \\
\hline $\mathrm{S} 2=\partial_{v} \partial_{i} \beta_{i}$ & $\mathrm{~V} 2_{i}=\partial_{v}^{2} \beta_{i}$ & $\mathrm{~T} 2_{i j}=\partial_{(i} l_{j)}$ \\
\hline $\mathrm{S} 3=\partial^{2} m$ & $\mathrm{~V} 3_{i}=\partial_{v} l_{i}$ & $\mathrm{~T} 3_{i j}=\partial_{v} \sigma_{i j}$ \\
\hline $\mathrm{ST} 1=\partial_{v} \beta_{i} \partial_{v} \beta_{i}$ & $\mathrm{~V} 4_{i}=\frac{9}{5} \partial_{j} \sigma_{j i}-\partial^{2} \beta_{i}$ & $\mathrm{TT}_{i j}=\partial_{v} \beta_{i} \partial_{v} \beta_{j}-\frac{1}{3} \mathrm{ST} 1 \delta_{i j}$ \\
\hline $\mathrm{ST} 2=l_{i} \partial_{v} \beta_{i}$ & $\mathrm{~V} 5_{i}=\partial^{2} \beta_{i}$ & $\mathrm{TT} 2_{i j}=l_{(i} \partial_{v} \beta_{j)}-\frac{1}{3} \mathrm{ST} 2 \delta_{i j}$ \\
\hline $\mathrm{ST} 3=\left(\partial_{i} \beta_{i}\right)^{2}$ & $\mathrm{VT}_{i}=\frac{1}{3}\left(\partial_{v} \beta_{i}\right)\left(\partial_{j} \beta^{j}\right)$ & $\mathrm{TT} 3_{i j}=2 \epsilon_{k l(i} \partial_{v} \beta^{k} \partial_{j)} \beta^{l}+\frac{2}{3} \mathrm{ST} 2 \delta_{i j}$ \\
\hline $\mathrm{ST} 4=l_{i} l^{i}$ & $\mathrm{VT} 2_{i}=-\epsilon_{i j k} l^{j} \partial_{v} \beta^{k}$ & $\mathrm{TT} 4_{i j}=\partial_{k} \beta^{k} \sigma_{i j}$ \\
\hline $\mathrm{ST} 5=\sigma_{i j} \sigma^{i j}$ & $\mathrm{VT} 3_{i}=\sigma_{i j} \partial_{v} \beta^{j}$ & $\mathrm{TT} 5_{i j}=l_{i} l_{j}-\frac{1}{3} \mathrm{ST} 4 \delta_{i j}$ \\
\hline $\mathrm{QS} 1=\partial_{v}^{2} q$ & $\mathrm{VT} 4_{i}=l_{i} \partial_{j} \beta^{j}$ & $\mathrm{TT} 6_{i j}=\sigma_{i k} \sigma_{j}^{k}-\frac{1}{3} \operatorname{ST} 5 \delta_{i j}$ \\
\hline $\mathrm{QS} 2=\partial_{i} \partial_{i} q$ & $\mathrm{VT} 5_{i}=\sigma_{i j} l^{j}$ & $\mathrm{TT} 7_{i j}=2 \epsilon_{m n(i} l^{m} \sigma_{j)}^{n}$ \\
\hline $\mathrm{QS} 3=l_{i} \partial_{i} q$ & $\mathrm{QV} 1_{i}=\partial_{i} \partial_{v} q$ & $\mathrm{QT}_{i j}=\partial_{i} \partial_{j} q-\frac{1}{3} \mathrm{QS} 2 \delta_{i j}$ \\
\hline $\mathrm{QS} 4=\left(\partial_{i} q\right)^{2}$ & $\mathrm{QV} 2_{i}=\partial_{i} q \partial_{k} \beta^{k}$ & $\mathrm{QT} 2_{i j}=\partial_{(i} q l_{j)}-\frac{1}{3} \mathrm{QS} 3 \delta_{i j}$ \\
\hline \multirow[t]{3}{*}{$\mathrm{QS} 5=\left(\partial_{i} q\right)\left(\partial_{v} \beta_{i}\right)$} & $\mathrm{QV} 3_{i}=\epsilon_{i j k} \partial_{j} l_{k}$ & $\mathrm{QT}_{i j}=\partial_{(i} q \partial_{j)} q-\frac{1}{3} \mathrm{QS} 4 \delta_{i j}$ \\
\hline & $\mathrm{QV}_{i}=\sigma_{i j} \partial_{j} q$ & $\mathrm{QT} 4_{i j}=\partial_{(i} q \partial_{v} \beta_{j)}-\frac{1}{3} \mathrm{QS} 5 \delta_{i j}$ \\
\hline & $\mathrm{QV} 5_{i}=\epsilon_{i j k} \partial_{v} \beta_{j} \partial_{k} q$ & $\mathrm{QT}_{i j}=\epsilon_{(i k m} \partial_{k} q \sigma_{m j)}$ \\
\hline
\end{tabular}

Table 1. An exhaustive list of two derivative terms in made up from the mass, charge and velocity fields. In order to present the results economically, we have dropped the superscript on the velocities $\beta_{i}$ charge $q$ and the mass $m$, leaving it implicit that these expressions are only valid at second order in the derivative expansion.

terms which are listed in table 1 . These relations are

$$
\begin{aligned}
\mathrm{S} 1 & =\frac{\mathrm{S} 3}{3}-\frac{8 m}{3} \mathrm{ST} 1+\frac{16 m}{9} \mathrm{ST} 3-\frac{2 m}{3} \mathrm{ST} 4+\frac{4 m}{3} \mathrm{ST} 5 \\
\mathrm{~S} 2 & =-\frac{1}{4 m} \mathrm{~S} 3+4 \mathrm{ST} 1+\frac{1}{2} \mathrm{ST} 4-\mathrm{ST} 5 \\
\mathrm{QS} 1 & =q(-\mathrm{ST} 1-\mathrm{S} 2+\mathrm{ST} 3)-\mathrm{QS} 5 \\
\mathrm{~V} 1_{i}= & m\left(-\frac{40}{9} \mathrm{~V} 4_{i}-\frac{4}{9} \mathrm{~V} 5_{i}+\frac{56}{3} \mathrm{VT} 1_{i}+\frac{4}{3} \mathrm{VT} 2_{i}+\frac{8}{3} \mathrm{VT} 3_{i}\right) \\
\mathrm{V} 2_{i}= & \frac{10}{9} \mathrm{~V} 4_{i}+\frac{1}{9} \mathrm{~V} 5_{i}-\frac{2}{3} \mathrm{VT} 1_{i}+\frac{1}{6} \mathrm{VT} 2_{i}-\frac{5}{3} \mathrm{VT} 3_{i} \\
\mathrm{~V} 3_{i}= & -\frac{1}{3} \mathrm{VT} 4_{i}+\mathrm{VT} 5_{i} \\
\mathrm{QV} 1_{i}= & -q\left(\frac{10}{3} \mathrm{~V} 4_{i}+\frac{1}{2}\left(\mathrm{VT} 2_{i}+2 \mathrm{VT} 1_{i}+2 \mathrm{VT} 3_{i}\right)+\frac{1}{3} \mathrm{~V} 5_{i}\right) \\
& -\mathrm{QV} 2_{i}-\frac{1}{2}\left(2 \mathrm{QV} 4_{i}+\mathrm{QV} 3_{i}+\frac{2}{3} \mathrm{QV} 2_{i}\right) \\
\mathrm{T} 1_{i j}= & -4 m\left(\mathrm{~T} 3_{i j}+\frac{1}{4} \mathrm{TT}_{i j}-4 \mathrm{TT} 1_{i j}+\frac{1}{3} \mathrm{TT} 4_{i j}+\mathrm{TT} 6_{i j}\right)
\end{aligned}
$$

With these relation between the source terms we will now solve the Einstein equations and 
Maxwell equations to find out the constraint and dynamical equations at second order in derivative expansion. As in the first order calculations we shall perform this seperately in various sectors denoting different representation of the boundary rotation group $\mathrm{SO}(3)$.

\subsection{Scalars of $\mathrm{SO}(3)$ at second order}

We parametrise the metric and the gauge field as follows

$$
\begin{aligned}
\sum_{i} g_{i i}^{(2)}(r) & =3 r^{2} h_{2}(r), \\
g_{v v}^{(2)}(r) & =\frac{k_{2}(r)}{r^{2}} \\
g_{v r}^{(2)}(r) & =-\frac{3}{2} h_{2}(r) \\
A_{v}^{(2)}(r) & =-\frac{\sqrt{3} w_{2}(r)}{2 r^{2}} .
\end{aligned}
$$

Now we intend to solve for the functions $h_{2}(r), k_{2}(r)$ and $w_{2}(r)$.

Constraint equations. As we have already explained, there are three constraint equations. First two come from Einstein equations (eq. (3.5) and 3.5) and the third one comes from Maxwell equations (eq. (3.7)). The first constrain from Einstein equations gives

$$
\partial_{v} m^{(1)}=\frac{2}{3} R^{3} \mathrm{ST} 5
$$

Second constraint implies relation between $k_{2}(r)$ and $h_{2}(r)$. This constraint equation is given by

$$
-m_{0} h_{2}^{\prime}(r)+3 r^{4} h_{2}^{\prime}(r)+12 r^{3} h_{2}(r)-k_{2}^{\prime}(r)+\frac{4 q_{0} w_{2}(r)}{r^{3}}-\frac{2 q_{0} w_{2}^{\prime}(r)}{r^{2}}=S_{C},
$$

where the source term $S_{C}$ is given in appendix B.

The constraint relation coming from Maxwell equations is given by

$$
\begin{aligned}
\partial_{v} q^{(1)}= & -\frac{3 q_{0}\left(R^{4}+m_{0}\right)}{16 m_{0}^{2} R} \mathrm{~S} 3+\frac{\left(R^{4}+m_{0}\right)}{4 m_{0} R} \mathrm{QS} 2-\frac{6 \sqrt{3} q_{0}{ }^{2} \kappa}{m_{0}} \mathrm{ST} 2 \\
& -\frac{\left(m_{0}-11 R^{4}\right)}{4 m_{0} R} \mathrm{QS} 5-\frac{2 \sqrt{3} q_{0} \kappa}{m_{0}} \mathrm{QS} 3-\frac{q_{0}}{4 m_{0} R^{3}} \mathrm{QS} 4+\frac{9 q_{0}\left(3 R^{4}+m_{0}\right)}{4 m_{0} R} \mathrm{ST} 1
\end{aligned}
$$

Dynamical equations and their solutions. The Dynamical Equations in the scalar sector (coming from the Einstein equation $E_{r r}=0$ ) is given by

$$
r h_{2}^{\prime \prime}(r)+5 h_{2}^{\prime}(r)=S_{h}
$$

The source term $S_{h}$ is explicitly given in appendix B.

The second dynamical scalar equation, which comes form the Maxwell equations $(M(r)=0)$, is given by

$$
-6 q_{0} h_{2}^{\prime}(r)+r w_{2}^{\prime \prime}(r)-w_{2}^{\prime}(r)=S_{M}(r) .
$$

The explicit form of the source term $S_{M}(r)$ is again given in appendix B. 
The source terms have the same large $r$ behavior as uncharged case (see [1]) because the charge dependent terms (leading) are more suppressed than that of charge independent terms. So one can follow the same procedure to obtain the solution for $h_{2}(r)$ and $k_{2}(r)$. Here we present the result schematically. Firstly, we solve equation (4.11) for the function $h_{2}(r)$; we obtain

$$
h_{2}(r)=\int\left(\frac{1}{r^{5}}\left(\int\left(r^{4} S_{h}(r)\right) d r+C_{h}^{(1)}\right)\right) d r+C_{h}^{(2)},
$$

where $C_{h}^{(1)}$ and $C_{h}^{(2)}$ are the constants of integration. We then plug in this solution for $h_{2}(r)$ in to (4.12). Solving the resultant equation for the $w_{2}$ we obtain,

$$
w_{2}(r)=\int\left(r\left(\int\left(\frac{1}{r^{2}} S_{w}(r)\right) d r+C_{w}^{(1)}\right)\right) d r+C_{w}^{(2)}
$$

where again $C_{w}^{(1)}$ and $C_{w}^{(2)}$ are integration constants, and the function $S_{w}(r)$ is

$$
S_{w}(r)=S_{M}(r)+6 q_{0} h_{2}^{\prime}(r) .
$$

Finally, we substitute the functions $h_{2}(r)$ and $w_{2}(r)$ solved above, in to (4.9) to obtain the following equation for $k_{2}(r)$

$$
k_{2}^{\prime}(r)=\left(3 r^{4}-m_{0}\right) h_{2}^{\prime}(r)+12 r^{3} h_{2}(r)+\frac{4 q_{0}}{r^{3}} w_{2}(r)-\frac{2 q_{0}}{r^{2}} w_{2}^{\prime}(r)-S_{C} \equiv S_{k}(r) .
$$

This equation can be easily integrated to obtain

$$
k_{2}(r)=\int S_{k}(r) d r+C_{k},
$$

$C_{k}$ being the integration constant. All the integration constants in the above solutions are obtained by imposing regularity at the horizon and normalizability of the functions, just as in the first order computation.

\subsection{Vectors of $\mathrm{SO}(3)$ at second order}

As given in (4.1) and (4.2), in this sector we parametrize ${ }^{10}$ the metric, and the gauge field respectively in the following way

$$
\begin{aligned}
g_{v i} & =6 r^{2} j_{i}^{(2)}(r) \\
A_{i}^{(2)} & =\frac{\sqrt{3}}{2} r^{2} g_{i}^{(2)}(r) .
\end{aligned}
$$

Constraint equations. In this sector, the constraint equation comes only from the Einstein equations (3.21). This constraint relation is give by

$$
\begin{aligned}
\partial_{i} m^{(1)}= & \frac{10 R^{3}}{9} \mathrm{~V} 4_{i}+\frac{10 R^{3}}{9} \mathrm{~V} 5_{i}+\frac{10 R^{3}}{3} \mathrm{VT} 1_{i}-\frac{5 R^{3}}{6} \mathrm{VT} 2_{i} \\
& +\frac{6 q_{0} R}{m_{0}-3 R^{4}} \mathrm{QV} 4_{i}-\frac{\left(21 R^{7}-43 m_{0} R^{3}\right)}{3\left(m_{0}-3 R^{4}\right)} \mathrm{VT} 3_{i} .
\end{aligned}
$$

\footnotetext{
${ }^{10}$ Note that the parametrization of the gauge field at this order is different from the one used for the scalar sector.
} 
Dynamical equations and their solutions. There are two vector dynamical equations. The first equation comes from Einstein equation and is given by

$$
q_{0} r g_{i}^{(2)^{\prime}}(r)+5 q_{0} g_{i}^{(2)}(r)+r j_{i}^{(2)^{\prime \prime}}(r)+5 j_{i}^{(2)^{\prime}}(r)=\left(S_{E}^{\mathrm{vec}}\right)_{i}(r),
$$

where $\left(S_{E}^{\mathrm{vec}}\right)_{i}(r)$ is the source terms given in the appendix C. The second dynamical equation comes from Maxwell equation and is given by

$$
\begin{aligned}
\sqrt{3}\left(-m_{0} r^{4} g_{i}^{(2)}(r)\right. & +q_{0}^{2} r^{2} g_{i}^{(2)^{\prime \prime}}(r)+r^{8} g_{i}^{(2)^{\prime \prime}}(r)+g_{i}^{(2)^{\prime}}(r)\left(-9 m_{0} r^{3}+7 q_{0}^{2} r+13 r^{7}\right) \\
& \left.+5 g_{i}^{(2)}(r)\left(-3 m_{0} r^{2}+q_{0}^{2}+7 r^{6}\right)+12 q_{0} j_{i}^{(2)^{\prime}}(r)\right)=\left(S_{M}^{\mathrm{vec}}\right)_{i}(r)
\end{aligned}
$$

where $\left(S_{M}^{\mathrm{vec}}\right)_{i}(r)$ is the other source term the explicit form of which is also given in the appendix C. The sources $\left(S_{M}^{\mathrm{vec}}\right)_{i}(r)$ and $\left(S_{E}^{\mathrm{vec}}\right)_{i}(r)$ are expressed in terms of the weyl invariant quantities $\left(W_{v}\right)_{i}^{m}$ which are defined in appendix A. We can now solve equation (4.19) for the function $g_{i}^{(2)}(r)$ to obtain

$$
g_{i}^{(2)}(r)=-\frac{j_{i}^{(2)^{\prime}}(r)}{q_{0}}+\frac{\left(W_{v}\right)_{i}^{1}+\left(W_{v}\right)_{i}^{2}}{6 q_{0} r^{3}}-\left(\frac{1}{q_{0} r^{5}}\right) \int_{r}^{\infty} x^{4}\left(\left(S_{E}^{\mathrm{vec}}\right)_{i}(r)-\frac{\left(W_{v}\right)_{i}^{1}+\left(W_{v}\right)_{i}^{2}}{3 x^{3}}\right) d x
$$

where the integrating constant has been chosen by the normalizability condition. Plugging in this solution in to (4.20) we obtain the following effective equation for $j_{i}^{(2)}(r)$

$$
\frac{d}{d r}\left(\frac{1}{r} \frac{d}{d r}\left(r^{7}\left(V^{(0)}(r)\right)^{2} \frac{d}{d r}\left(\frac{1}{V^{(0)}(r)} j_{i}^{(2)}(r)\right)\right)\right)+S_{i}(r)=0
$$

where

$$
\begin{aligned}
S_{i}(r)= & \left(-\frac{1}{\sqrt{3} r^{2}}\right)\left(\sqrt { 3 } \left(r\left(m_{0}\left(R^{2}-r^{2}\right)+r^{6}-R^{6}\right)\left(S_{E}^{\mathrm{vec}}\right)_{i}^{\prime}(r)\right.\right. \\
& \left.\left.+\left(S_{E}^{\mathrm{vec}}\right)_{i}(r)\left(m_{0}\left(R^{2}-3 r^{2}\right)+7 r^{6}-R^{6}\right)\right)-\sqrt{R^{2}\left(m_{0}-R^{4}\right)}\left(S_{M}^{\mathrm{vec}}\right)_{i}(r)\right) .
\end{aligned}
$$

Finally, the solution to the equation (4.22) is given by

$$
\begin{aligned}
j_{i}^{(2)}(r)= & -V^{(0)}(r) \int_{r}^{\infty} \frac{1}{x^{7}\left(V^{(0)}(x)\right)^{2}}\left(\int_{x}^{\infty} y \int_{y}^{\infty} S_{i}^{r e g}(z) d z d y\right) d x \\
& -V^{(0)}(r) \int_{r}^{\infty} \frac{1}{x^{7}\left(V^{(0)}(x)\right)^{2}}\left[C_{i}^{(j)}-\frac{1}{3\left(m_{0} 3 R^{2}\right)} 3 R^{7}\left(\left(\left(W_{v}\right)_{i}^{1}+\left(W_{v}\right)_{i}^{4}\right) x\right.\right. \\
& \left.\left.-m_{0} R^{3}\left(\left(W_{v}\right)_{i}^{1}+3\left(W_{v}\right)_{i}^{4}\right) x-\frac{1}{2} m_{0}\left(\left(W_{v}\right)_{i}^{1}+\left(W_{v}\right)_{i}^{2}\right) x^{4}+\frac{3}{2} R^{4}\left(\left(W_{v}\right)_{i}^{1}+\left(W_{v}\right)_{i}^{2}\right) x^{4}\right)\right] d x,
\end{aligned}
$$

where again for convenience we have defined

$$
S_{i}^{r e g}(z)=\frac{R^{3}\left(m_{0}\left(\left(W_{v}\right)_{i}^{1}+3\left(W_{v}\right)_{i}^{4}\right)-3 R^{4}\left(\left(W_{v}\right)_{i}^{1}+\left(W_{v}\right)_{i}^{4}\right)\right)}{3 z^{2}\left(m_{0}-3 R^{4}\right)}-S_{i}(z)-\frac{4}{3} z\left(\left(W_{v}\right)_{i}^{1}+\left(W_{v}\right)_{i}^{2}\right) .
$$


The constant $C_{i}^{(j)}$ is determined by the regularity at horizon and is given by

$$
\begin{aligned}
C_{i}^{(j)}= & -\frac{1}{12 m_{0}\left(m_{0}-3 R^{4}\right)}\left(R ^ { 4 } \left(m_{0}^{2}\left(9\left(W_{v}\right)_{i}^{1}+4\left(W_{v}\right)_{i}^{2}+15\left(W_{v}\right)_{i}^{4}\right)\right.\right. \\
& \left.-6 m_{0} R^{4}\left(6\left(W_{v}\right)_{i}^{1}+3\left(W_{v}\right)_{i}^{2}+4\left(W_{v}\right)_{i}^{4}\right)+9 R^{8}\left(3\left(W_{v}\right)_{i}^{1}+2\left(W_{v}\right)_{i}^{2}+\left(W_{v}\right)_{i}^{4}\right)\right) \\
& \left.-9 R^{2}\left(m_{0}^{2}-4 m_{0} R^{4}+3 R^{8}\right)\left(\int_{R}^{\infty} S_{i}^{\mathrm{reg}}(x) d x\right)+6 m_{0}\left(m_{0}-3 R^{4}\right) \int_{R}^{\infty} y^{2} S_{i}^{\mathrm{reg}}(y) d y\right),
\end{aligned}
$$

We now have to plug in the source terms (given in appendix C) and perform the integrals to write the solutions explicitly. Since such explicit solution would be very complicated, we do not provide it here. Nevertheless, from the above solution we extract the boundary charge current as we explicate in the following section.

\subsection{Boundary charge current at second order}

The charge current at second order in derivative expansion is given by

$$
J_{\mu}^{(2)}=\lim _{r \rightarrow \infty} \frac{r^{2} A_{\mu}^{(2)}}{8 \pi G_{5}}
$$

The gauge field perturbation at this order is parametrised by the function $g_{i}^{(2)}(r)$. Thus to obtain the charge current density we have to consider the asymptotic limit (i.e. the $r \rightarrow \infty$ limit) of the function $g_{i}^{(2)}(r)$. This function is given by (4.21). The function $j_{i}^{(2)}(r)$ in that equation is in turn given by (4.24).

If we carefully extract the coefficient of the $1 / r^{2}$ term in the $r \rightarrow \infty$ limit of the gauge field (using the equation referred to in the last paragraph) we find that the charge current is given by

$$
J_{i}^{(2)}=\frac{m_{0}\left(W_{v}\right)_{i}^{2}-6 C_{i}^{(j)}}{4 \sqrt{3} \sqrt{R^{2}\left(m_{0}-R^{4}\right)}},
$$

the constant $C_{i}^{(j)}$ being given by the equation (4.26). Plugging in the sources in to equation (4.26) and performing the integrations we find

$$
J_{i}^{(2)}=\left(\frac{1}{8 \pi G_{5}}\right) \sum_{l=1}^{5} \mathcal{C}_{l}\left(W_{v}\right)_{i}^{l}
$$


where the coefficients of the Weyl invariant terms $\left(W_{v}\right)_{i}^{l}$ are given by ${ }^{11}$

$$
\begin{aligned}
& \mathcal{C}_{1}=\frac{3 \sqrt{3} R \sqrt{M-1}}{8 M}, \\
& \mathcal{C}_{2}=\frac{\sqrt{3} R(M-1)^{3 / 2}}{4 M^{2}}, \\
& \mathcal{C}_{3}=-\frac{3 R \kappa(M-1)}{2 M^{2}}, \\
& \mathcal{C}_{4}=\frac{1}{4} \sqrt{3} R \sqrt{M-1} \log (2)+\mathcal{O}(M-1), \\
& \mathcal{C}_{5}=-\frac{\sqrt{3} R \sqrt{M-1}\left(M^{2}-48(M-1) \kappa^{2}+3\right)}{16 M^{2}} .
\end{aligned}
$$

We have expressed the above results in terms of the parameters $M$ and $R$ with $M=m_{0} / R^{4}$.

\subsection{Tensors of $\mathrm{SO}(3)$ at second order}

We now consider the tensor modes at second order. Following the first order calculations we pametrize the traceless symmetric tensor components of the second order metric by the function $\alpha_{i j}^{(2)}(r)$ such that

$$
g_{i j}^{(2)}=r^{2} \alpha_{i j}^{(2)}(r)
$$

In this sector there are no constraint equations. However, there is a dynamical equation which we solve in the following subsection.

Dynamical equations and their solutions. The $i j$-component of the Einstein equation gives the dynamical equation for $\alpha_{i j}^{(2)}(r)$ which is similar to (3.33). However the source term of the differential equation is modified in the second order. Thus, at second order this equation is given by

$$
-\frac{1}{2 r} \frac{d}{d r}\left(\frac{1}{r}\left(q_{0}^{2}-m_{0} r^{2}+r^{6}\right) \frac{d}{d r} \alpha_{i j}^{(2)}(r)\right)=\mathbf{T}_{i j}(r),
$$

where we write the source in terms of weyl-covariant quantities as follows

$$
\mathbf{T}_{i j}(r)=\sum_{l=1}^{9} \tau_{l}(r) W T_{i j}^{(l)} .
$$

We define the weyl-covariant terms $W T_{i j}^{(l)}$ in appendix A. The coefficients $\tau_{l}(r)$ of these weyl-covariant terms are given in appendix D.

The solution to (4.32) which is regular at the outer horizon and normalizable at infinity is given by

$$
\alpha_{i j}^{(2)}(r)=\int_{r}^{\infty}\left(\left(\frac{\xi}{q_{0}^{2}-m_{0} \xi^{2}+\xi^{6}}\right) \int_{1}^{\xi}\left(2 \zeta \mathbf{T}_{i j}(\zeta)\right) d \zeta\right) d \xi
$$

We need to plug in the source from appendix D in to the above equation and perform the integrals to obtain an explicit answer. However, as in the second order vector sector this

\footnotetext{
${ }^{11}$ All these coefficients perfectly match with the corresponding coefficients in version 4 of [61].
} 
turns out to be very complicated in general and therefore we do not produce it here. The transport coefficients, however, of the boundary stress tensor at second order in derivative expansion may be obtained only by knowing the function $\alpha_{i j}^{(2)}(r)$ asymptotically (near the boundary). In the next subsection, we compute this boundary stress tensor.

\subsection{Boundary stress tensor at second order}

As mentioned earlier in subsection 3.5, the AdS/CFT prescription for obtaining the boundary stress tensor from the bulk metric is given by

$$
T_{\nu}^{\mu}=-\frac{1}{8 \pi G_{5}} \lim _{r \rightarrow \infty}\left(r^{4}\left(K_{\nu}^{\mu}-\delta_{\nu}^{\mu}\right)\right),
$$

where $K_{\nu}^{\mu}$ is the extrinsic curvature normal to the constant r surface. Now, as is apparent from the formula, we need to know the asymptotic expansion of the metric perturbation $\alpha_{i j}^{(2)}(\rho)$ in order to obtain the stress tensor. The asymptotic expansion of the solution (4.34) for $\alpha_{i j}^{(2)}(\rho)$ is given by

$$
\alpha_{i j}^{(2)}(\rho)=\frac{1}{r^{2}}\left(W T_{i j}^{(3)}-\frac{1}{2} W T_{i j}^{(2)}-\frac{1}{4} W T_{i j}^{(4)}\right)+\frac{1}{4 r^{4}} \sum_{l=1}^{9} \mathcal{N}_{l} W T_{i j}^{(l)}+\mathcal{O}\left(\frac{1}{r^{5}}\right),
$$

The leading term of this asymptotic expansion gives divergent contributions to the stress tensor which are canceled by divergence arising from the expansion of $g^{(0)}+g^{(1)}$ up to second order.

On plugging in this asymptotic solution for the metric in to the formula (4.35) we obtain

$$
T_{\mu \nu}=\left(\frac{1}{16 \pi G_{5}}\right) \sum_{l=1}^{9} \mathcal{N}_{l} W T_{\mu \nu}^{(l)}
$$

with $\mathcal{N}_{l}$ being the transport coefficients at second order in derivative expansion. These transport coefficients are given by

$$
\begin{aligned}
& \left.\mathcal{N}_{1}=R^{2}\left(\frac{M}{\sqrt{4 M-3}} \log \left(\frac{3-\sqrt{4 M-3}}{3+\sqrt{4 M-3}}\right)+2\right)\right) \\
& \mathcal{N}_{2}=-\frac{M R^{2}}{2 \sqrt{4 M-3}} \log \left(\frac{3-\sqrt{4 M-3}}{\sqrt{4 M-3}+3}\right) \\
& \mathcal{N}_{3}=2 R^{2} \\
& \mathcal{N}_{4}=\frac{R^{2}}{M}(M-1)\left(12(M-1) \kappa^{2}-M\right) \\
& \mathcal{N}_{5}=-\frac{(M-1) R^{2}}{2 M} \\
& \mathcal{N}_{6}=\frac{1}{2}(M-1) R^{2}(\log (8)-1)+\mathcal{O}\left((M-1)^{2}\right), \\
& \mathcal{N}_{7}=\frac{\sqrt{3}(M-1)^{3 / 2} R^{2} \kappa}{M} \\
& \mathcal{N}_{8}=0 \\
& \mathcal{N}_{9}=0
\end{aligned}
$$




\section{Discussion}

In this paper, we have computed the metric dual to a fluid with a globally conserved charge and used that to find the energy-momentum tensor and the charge current in arbitrary fluid configurations to second order in the boundary derivative expansion.

Note that the corresponding construction of the bulk metric dual to an uncharged fluid flow was characterized by a great deal of universality. This universality had its origin in the fact that every two derivative theory of gravity (interacting with other fields) that admits $A d S_{5}$ as a solution, also admits a consistent truncation to the equations of Einstein gravity with a negative cosmological constant. This universality does not extend to Einstein Maxwell system. The possibility of extending the Einstein Maxwell system by a Chern Simon's term of arbitrary coefficient, accounted for in this paper, is an illustration of the reduced universality of our calculations.

We have seen that a nonzero value for the coefficient of the Chern-Simons in the bulk leads to an interesting dual hydrodynamic effect (recall that this coefficient is indeed nonzero in strongly coupled $\mathcal{N}=4$ Yang Mills ). At first order in the derivative expansion we find that the charge current has a term proportional to $l^{\alpha} \equiv \epsilon^{\mu \nu \lambda \alpha} u_{\mu} \partial_{\nu} u_{\lambda}$ in addition to the more familiar Fick type diffusive term. It would be interesting to reproduce this term from a computation directly in the boundary. In more general terms, it would be interesting to gain better intuition for effect induced by the bulk Chern-Simons term on boundary dynamics.

We have already remarked on the fact that the pseudo-tensor terms appearing in the stress tensor and the charge current solve an old puzzle raised in [45] regarding the fluidgravity correspondence in large rotating AdS charged black holes. The discrepancy found by the authors of [45] between the fluid dynamical predictions and the known thermodynamics of rotating black holes is basically resolved, once the qualitatively new effects due to the bulk Chern-Simons interaction on hydrodynamics is taken into account. We will reserve a deeper analysis of this issue along with a detailed comparison of our second order fluid dynamical metric and gauge field with charged black hole solutions to future work.

Though we have not yet worked out explicitly the position of the event horizon in our metric solutions, it is plausible that the analysis of [2] can be easily extended to the case of metrics with a global conserved charge(at least for the non-extremal case). This expectation, however has to be confirmed by an explicit computation. Further, it would be interesting to derive an entropy current for the charged fluid following the proposal outlined in [2].

In the bulk of our work we have refrained from commenting on the fluids near extremality. The preliminary analysis in [45] suggests that hydrodynamics would be a valid description at least for some class of extremal solutions. Unfortunately, we have not been able to shed more light on this issue in the present work - the perturbative metric and the gauge field we have obtained are ill-behaved at the horizon in the near-extremal limit. In fact, even in the non-extremal case considered by us here, the components of our solution diverge at the inner horizon (this divergence is however shielded by the outer horizon). We leave unanswered the question of whether this is a co-ordinate artifact, since this is 
a question which requires a detailed analysis of the causal structure of our solutions. If this divergence is physical in the extremal case, it might have very interesting implications. This is indeed one of the most pressing questions opened up by the present work and it would be interesting to understand the extremal limit of our solutions more clearly.

\section{Acknowledgement}

The authors would like to thank Shiraz Minwalla for suggesting this problem and providing guidance throughout the project. We would also like to thank Veronica Hubeny, Mukund Rangamani and Sandip Trivedi for helpful discussions during the project. NB and SD would like to thank D. Astefanesei, R. Gopakumar, D. Jatkar and A. Sen for discussions. We also thank all the students of Theory Physics student's room in TIFR for help and several useful discussions. We would like to acknowledge the organizers of the Monsoon Workshop on String theory at the Tata Institute of Fundamental Research, Mumbai(organized by the International centre for Theoretical Sciences) during which a major part of this work was done. NB, JB and SD would like to thank the organisers of AdS/QCD school, 2008 at ICTP, where the part of the work was done. PS would like to thank the Tata Institute of Fundamental Research for its hospitality and for partial support during the completion of this work. PS was supported by Polish Ministry of Science and Information Technologies grant 1P03B04029 (2005-2008).NB, SB, JB, SD and RL would like to thank people of India for their generous support to fundamental research.

\section{A Charged conformal fluids and Weyl covariance}

Consider the hydrodynamic limit of a $3+1$ dimensional CFT with one global conserved charge. The Weyl covariance of the CFT translates into the Weyl covariance of its hydrodynamics. In turn, this implies that the metric dual to fluid configurations of the CFT under consideration should also be invariant under boundary Weyl-transformations $[2,3,6]$.

In this section, we use the manifestly Weyl-covariant formalism introduced in [6] to examine the constraints that Weyl-covariance imposes on the conformal hydrodynamics and its metric dual. We begin by introducing a Weyl-covariant derivative acting on a general tensor field $Q_{\nu \ldots}^{\mu \ldots}$ with weight $w$ (by which we mean that the tensor field transforms as $Q_{\nu \ldots}^{\mu \ldots}=e^{-w \phi} \tilde{Q}_{\nu \ldots}^{\mu \ldots}$ under a Weyl transformation of the boundary metric $g_{\mu \nu}=e^{2 \phi} g_{\mu \nu}$ )

$$
\begin{aligned}
\mathcal{D}_{\lambda} Q_{\nu \ldots}^{\mu \ldots} \equiv & \nabla_{\lambda} Q_{\nu \ldots}^{\mu \ldots}+w \mathcal{A}_{\lambda} Q_{\nu \ldots}^{\mu \ldots} \\
& +\left[g_{\lambda \alpha} \mathcal{A}^{\mu}-\delta_{\lambda}^{\mu} \mathcal{A}_{\alpha}-\delta_{\alpha}^{\mu} \mathcal{A}_{\lambda}\right] Q_{\nu \ldots}^{\alpha \ldots}+\ldots \\
& -\left[g_{\lambda \nu} \mathcal{A}^{\alpha}-\delta_{\lambda}^{\alpha} \mathcal{A}_{\nu}-\delta_{\nu}^{\alpha} \mathcal{A}_{\lambda}\right] Q_{\alpha \ldots}^{\mu \ldots}-\ldots
\end{aligned}
$$

where the Weyl-connection $\mathcal{A}_{\mu}$ is related to the fluid velocity $u^{\mu}$ via the relation

$$
\mathcal{A}_{\mu}=u^{\lambda} \nabla_{\lambda} u_{\mu}-\frac{\nabla_{\lambda} u^{\lambda}}{3} u_{\mu}
$$

We can now use this Weyl-covariant derivative to enumerate all the Weyl-covariant scalars, transverse vectors (i.e, vectors that are everywhere orthogonal to the fluid velocity 
field $u^{\mu}$ ) and the transverse traceless tensors in the charged hydrodynamics that involve no more than second order derivatives. We will do this enumeration 'on-shell', i.e., we will enumerate those quantities which remain linearly independent even after the equations of motion are taken into account. Our discussion here will closely parallel the discussion in section 4.1 of [3] where a similar question was answered in the context of uncharged hydrodynamics coupled to a scalar with weight zero. However, we will use a slightly different basis of Weyl-covariant tensors which is more suited for purposes of this paper.

The basic fields in the charged hydrodynamics are the fluid velocity $u^{\mu}$ with weight unity, the fluid temperature $T$ with with weight unity and the chemical potential $\mu$ with weight unity. This implies that an arbitrary function of $\mu / T$ is Weyl-invariant and hence one could always multiply a Weyl-covariant tensor by such a function to get another Weylcovariant tensor. Hence, in the following list only linearly independent fields appear. To make contact with the conventional literature on hydrodynamics we will work with the charge density $n$ (with weight 3 ) rather than the chemical potential $\mu$.

At one derivative level, there are no Weyl invariant scalars or pseudo-scalars. The only Weyl invariant transverse vector is $n^{-1} P_{\mu}^{\nu} \mathcal{D}_{\nu} n$. Finally, the only Weyl-invariant transverse pseudo-vector $l_{\mu}$ and only one Weyl-invariant symmetric traceless transverse tensor $T \sigma_{\mu \nu}$.

At the two derivative level, there are five independent Weyl-invariant scalars ${ }^{12}$

$$
T^{-2} \sigma_{\mu \nu} \sigma^{\mu \nu}, \quad T^{-2} \omega_{\mu \nu} \omega^{\mu \nu}, \quad T^{-2} \mathcal{R}, \quad T^{-2} n^{-1} P^{\mu \nu} \mathcal{D}_{\mu} \mathcal{D}_{\nu} n \quad \text { and } \quad T^{-2} n^{-2} P^{\mu \nu} \mathcal{D}_{\mu} n \mathcal{D}_{\nu} n
$$

one Weyl-invariant pseudo-scalar $T^{-2} n^{-1} l^{\mu} \mathcal{D}_{\mu} n$ and four independent Weyl-invariant transverse vectors

$$
T^{-1} P_{\mu}^{\nu} \mathcal{D}_{\lambda}{\sigma_{\nu}}^{\lambda}, \quad T^{-1} P_{\mu}^{\nu} \mathcal{D}_{\lambda} \omega_{\nu}{ }^{\lambda}, \quad T^{-1} n^{-1}{\sigma_{\mu}}^{\lambda} \mathcal{D}_{\lambda} n \quad \text { and } \quad T^{-1} n^{-1} \omega_{\mu}{ }^{\lambda} \mathcal{D}_{\lambda} n
$$

and one Weyl-invariant transverse pseudo-vector $T^{-1} \sigma_{\mu \nu} l^{\nu}$.

There are eight Weyl-invariant symmetric traceless transverse tensors -

$$
\begin{aligned}
& u^{\lambda} \mathcal{D}_{\lambda} \sigma_{\mu \nu}, \quad \omega_{\mu}{ }^{\lambda} \sigma_{\lambda \nu}+\omega_{\nu}{ }^{\lambda} \sigma_{\lambda \mu}, \quad \sigma_{\mu}{ }^{\lambda} \sigma_{\lambda \nu}-\frac{P_{\mu \nu}}{3} \sigma_{\alpha \beta} \sigma^{\alpha \beta}, \quad \omega_{\mu}{ }^{\lambda} \omega_{\lambda \nu}+\frac{P_{\mu \nu}}{3} \omega_{\alpha \beta} \omega^{\alpha \beta}, \\
& n^{-1} \Pi_{\mu \nu}^{\alpha \beta} \mathcal{D}_{\alpha} \mathcal{D}_{\beta} n, \quad n^{-2} \Pi_{\mu \nu}^{\alpha \beta} \mathcal{D}_{\alpha} n \mathcal{D}_{\beta} n, \quad C_{\mu \alpha \nu \beta} u^{\alpha} u^{\beta} \quad \text { and } \quad \frac{1}{4} \epsilon^{\alpha \beta \lambda}{ }_{\mu} \epsilon^{\gamma \theta \sigma}{ }_{\nu} C_{\alpha \beta \gamma \theta} u_{\lambda} u_{\sigma} .
\end{aligned}
$$

where we have introduced the projection tensor $\Pi_{\mu \nu}^{\alpha \beta}$ which projects out the transverse traceless symmetric part of second rank tensors

$$
\Pi_{\mu \nu}^{\alpha \beta} \equiv \frac{1}{2}\left[P_{\mu}^{\alpha} P_{\nu}^{\beta}+P_{\nu}^{\alpha} P_{\mu}^{\beta}-\frac{2}{3} P^{\alpha \beta} P_{\mu \nu}\right]
$$

\footnotetext{
${ }^{12}$ We shall follow the notations of [6] in the rest of this section (except for the curvature tensors which differ by a sign from the curvature tensors in [6]). In particular, we recall the following definitions

$$
\begin{aligned}
\mathcal{R} & =R+6 \nabla_{\lambda} \mathcal{A}^{\lambda}-6 \mathcal{A}_{\lambda} \mathcal{A}^{\lambda} ; & \mathcal{D}_{\mu} u_{\nu} & =\sigma_{\mu \nu}+\omega_{\mu \nu} \\
\mathcal{D}_{\lambda} \sigma^{\mu \lambda} & =\nabla_{\lambda} \sigma^{\mu \lambda}-3 \mathcal{A}_{\lambda} \sigma^{\mu \lambda} ; & \mathcal{D}_{\lambda} \omega^{\mu \lambda} & =\nabla_{\lambda} \omega^{\mu \lambda}-\mathcal{A}_{\lambda} \omega^{\mu \lambda}
\end{aligned}
$$

Note that in a flat space-time, $R$ is zero but $\mathcal{R}$ is not. 
and $C_{\mu \nu \alpha \beta}$ is the boundary Weyl curvature tensor. Further, there are four Weyl-invariant symmetric traceless transverse pseudo-tensors

$$
\mathcal{D}_{(\mu} l_{\nu)}, \quad n^{-1} \Pi_{\mu \nu}^{\alpha \beta} l_{\alpha} \mathcal{D}_{\beta} n, \quad n^{-1} \epsilon_{(\mu}^{\alpha \beta \lambda} \sigma_{\nu) \lambda} u_{\alpha} \mathcal{D}_{\beta} n \quad \text { and } \quad \frac{1}{2} \epsilon_{\alpha \beta \lambda(\mu} C^{\alpha \beta}{ }_{\nu) \sigma} u^{\lambda} u^{\sigma} .
$$

We will now restrict ourselves to the case where the boundary metric is flat. In this case the last two tensors appearing in (A.6) and the last tensor appearing in (A.7) are identically zero whereas, contrary to what one might naively expect, the Weyl-covariantised Ricci scalar $\mathcal{R}$ would still be non-zero.

We will now relate the rest of the Weyl-covariant scalars, transverse vectors and symmetric, traceless transverse tensors listed above to the quantities appearing in the table 1.

There are six scalar/pseudo-scalar Weyl covariant combinations given by

$$
\begin{aligned}
& W_{s}^{1} \equiv \sigma_{\mu \nu} \sigma^{\mu \nu}=\mathrm{ST} 5 \\
& W_{s}^{2} \equiv \omega_{\mu \nu} \omega^{\mu \nu}=\frac{1}{2} \mathrm{ST} 4 \\
& W_{s}^{3} \equiv \mathcal{R}=14 \mathrm{ST} 1+\frac{2}{3} \mathrm{ST} 3-\mathrm{ST} 4+2 \mathrm{ST} 5-\frac{\mathrm{S} 3}{m} \\
& W_{s}^{4} \equiv n^{-1} P^{\mu \nu} \mathcal{D}_{\mu} \mathcal{D}_{\nu} n=\frac{1}{q}\left[\mathrm{QS} 2-\frac{3 q}{4 m} \mathrm{~S} 3+18 \mathrm{qST} 1+5 \mathrm{QS} 5\right] \\
& W_{s}^{5} \equiv n^{-2} P^{\mu \nu} \mathcal{D}_{\mu} n \mathcal{D}_{\nu} n=\frac{1}{q^{2}}\left[\mathrm{QS} 4+6 \mathrm{qQS} 5+9 \mathrm{q}^{2} \mathrm{ST} 1\right] \\
& W_{s}^{6} \equiv l^{\mu} \mathcal{D}_{\mu} q=\mathrm{QS} 3+3 \mathrm{qST} 2 .
\end{aligned}
$$

and five vector/pseudo-vector Weyl covariant combinations given by

$$
\begin{aligned}
\left(W_{v}\right)_{\mu}^{1} & \equiv P_{\mu}^{\nu} \mathcal{D}_{\lambda} \sigma_{\nu}{ }^{\lambda}=\frac{5 \mathrm{~V} 4}{9}+\frac{5 \mathrm{~V} 5}{9}+\frac{5 \mathrm{VT} 1}{3}-\frac{5 \mathrm{VT} 2}{12}-\frac{11 \mathrm{VT} 3}{6} \\
\left(W_{v}\right)_{\mu}^{2} & \equiv P_{\mu}^{\nu} \mathcal{D}_{\lambda} \omega_{\nu}{ }^{\lambda}=\frac{5 \mathrm{~V} 4}{3}-\frac{\mathrm{V} 5}{3}-\mathrm{VT} 1-\frac{\mathrm{VT} 2}{4}+\frac{\mathrm{VT} 3}{2} \\
\left(W_{v}\right)_{\mu}^{3} & \equiv l^{\lambda} \sigma_{\mu \lambda}=\mathrm{VT} 5 \\
\left(W_{v}\right)_{\mu}^{4} & \equiv n^{-1} \sigma_{\mu}{ }^{\lambda} \mathcal{D}_{\lambda} n=\frac{1}{q}[\mathrm{QV} 4+3 \mathrm{qVT} 3] \\
\left(W_{v}\right)_{\mu}^{5} & \equiv n^{-1} \omega_{\mu}{ }^{\lambda} \mathcal{D}_{\lambda} n=\frac{1}{2 q}[\mathrm{QV} 3+3 \mathrm{qVT} 2]
\end{aligned}
$$

In the tensor sector, there are nine Weyl-covariant combinations

$$
\begin{aligned}
W T_{\mu \nu}^{(1)} & =u^{\lambda} \mathcal{D}_{\lambda} \sigma_{\mu \nu}=T T 1+\frac{1}{3} T T 4+T 3 . \\
W T_{\mu \nu}^{(2)} & =-2\left(\omega^{\mu}{ }_{\lambda} \sigma^{\lambda \nu}+\omega^{\nu}{ }_{\lambda} \sigma^{\lambda \mu}\right)=T T 7 . \\
W T_{\mu \nu}^{(3)} & =\sigma^{\mu}{ }_{\lambda} \sigma_{\lambda \nu}-\frac{1}{3} P^{\mu \nu} \sigma^{\alpha \beta} \sigma_{\alpha \beta}=T T 6 . \\
W T_{\mu \nu}^{(4)} & =4\left(\omega^{\mu}{ }_{\lambda} \omega_{\lambda \nu}+\frac{1}{3} P^{\mu \nu} \omega^{\alpha \beta} \omega_{\alpha \beta}\right)=T T 5 . \\
W T_{\mu \nu}^{(5)} & =n^{-1} \Pi_{\mu \nu}^{\alpha \beta} \mathcal{D}_{\alpha} \mathcal{D}_{\beta} n \\
& =\frac{1}{q}\left[Q T 1+8 Q T 4+15 q T T 1+q T T 4+3 q T 3+3 q T T 6+\frac{3 q}{4} T T 5\right]
\end{aligned}
$$




$$
\begin{aligned}
W T_{\mu \nu}^{(6)} & =n^{-2} \Pi_{\mu \nu}^{\alpha \beta} \mathcal{D}_{\alpha} n \mathcal{D}_{\beta} n=\frac{1}{q^{2}}\left[Q T 3+6 q Q T 4+9 q^{2} T T 1\right] \\
W T_{\mu \nu}^{(7)} & =\mathcal{D}_{\mu} l_{\nu}+\mathcal{D}_{\nu} l_{\mu}=4 T T 2+2 T 2-T T 3 . \\
W T_{\mu \nu}^{(8)} & =n^{-1} \Pi_{\mu \nu}^{\alpha \beta} l_{\alpha} \mathcal{D}_{\beta} n=\frac{1}{q}[Q T 2+3 q T T 2] . \\
W T_{\mu \nu}^{(9)} & =n^{-1} \epsilon_{(\mu}^{\alpha \beta \lambda} \sigma_{\nu) \lambda} u_{\alpha} \mathcal{D}_{\beta} n=\frac{1}{q}\left[Q T 5-\frac{3}{2} q T T 2+\frac{3}{2} q T T 3\right] .
\end{aligned}
$$

\section{B Source terms in scalar sector: second order}

There are three source terms in scalar sector at second order $S_{k}(r), S_{h}(r)$ and $S_{M}(r)$. They are quite complicated functions. Here we provide the explicit form of these source terms in terms of weyl covariant quantities.

The source term $S_{k}$ is given by

$$
S_{C}=\sum_{i=1}^{6} s_{i}^{(C)} W_{s}^{i} .
$$

The Weyl covariant terms $W_{s}^{i}$ are given in appendix A. The functions $s_{i}^{(k)} \mathrm{s}$ are given by,

$$
\begin{aligned}
s_{1}^{(C)}= & \frac{r\left(4\left(m_{0}-3 r^{4}\right)\left(r^{2}+r R+R^{2}\right) F_{2}\left(\frac{r}{R}, \frac{m_{0}}{R^{4}}\right)+R\left(m_{0}(r+R)-2 R^{3}\left(r^{2}+r R+R^{2}\right)\right)\right)}{3 R(r+R)\left(-m_{0}+r^{4}+r^{2} R^{2}+R^{4}\right)} \\
s_{2}^{(C)}= & \frac{1}{3 m_{0}^{2} r^{7}}\left(-m_{0}^{3}\left(r^{4}+2 r^{2} R^{2}+36 R^{4} \kappa^{2}\right)+2 m_{0}^{2}\left(18 r^{4} R^{4} \kappa^{2}+r^{2} R^{6}+36 R^{8} \kappa^{2}\right)\right. \\
& \left.-36 m_{0} R^{8} \kappa^{2}\left(2 r^{4}+R^{4}\right)+36 r^{4} R^{12} \kappa^{2}\right) \\
s_{3}^{(C)}= & \frac{r}{3} \\
s_{4}^{(C)}= & \frac{2 r^{2}\left(m_{0}-R^{4}\right)\left(r F_{1}^{(1,0)}\left(\frac{r}{R}, \frac{m_{0}}{R^{4}}\right)+6 R F_{1}\left(\frac{r}{R}, \frac{m_{0}}{R^{4}}\right)\right)}{R^{6}} \\
s_{5}^{(C)}= & -\frac{1}{2 R^{16}\left(m_{0}-3 R^{4}\right)}\left(r ^ { 2 } ( m _ { 0 } - R ^ { 4 } ) \left(2 4 R ^ { 4 } F _ { 1 } ( \frac { r } { R } , \frac { m _ { 0 } } { R ^ { 4 } } ) \left(r ^ { 3 } \left(m_{0}^{2}-4 m_{0} R^{4}\right.\right.\right.\right. \\
& \left.+3 R^{8}\right) F_{1}^{(2,0)}\left(\frac{r}{R}, \frac{m_{0}}{R^{4}}\right)+11 r^{2} R\left(m_{0}^{2}-4 m_{0} R^{4}+3 R^{8}\right) F_{1}^{(1,0)}\left(\frac{r}{R}, \frac{m_{0}}{R^{4}}\right) \\
& \left.+6 m_{0} R^{7}-4 R^{11}\right)+r\left(r ^ { 2 } R ^ { 2 } \left(m_{0}^{2}\left(25 r^{2}-13 R^{2}\right)+m_{0}\left(-25 r^{6}-75 r^{2} R^{4}+52 R^{6}\right)\right.\right. \\
& \left.+75 r^{6} R^{4}-39 R^{10}\right) F_{1}^{(1,0)}\left(\frac{r}{R}, \frac{m_{0}}{R^{4}}\right)^{2}+r F_{1}^{(2,0)}\left(\frac{r}{R}, \frac{m_{0}}{R^{4}}\right)\left(4 R^{9}\left(m_{0}-R^{4}\right)\right. \\
& \left.-r^{3}\left(m_{0}^{2}\left(R^{2}-r^{2}\right)+m_{0}\left(r^{6}+3 r^{2} R^{4}-4 R^{6}\right)+3 R^{4}\left(R^{6}-r^{6}\right)\right) F_{1}^{(2,0)}\left(\frac{r}{R}, \frac{m_{0}}{R^{4}}\right)\right) \\
& +2 R F_{1}^{(1,0)}\left(\frac{r}{R}, \frac{m_{0}}{R^{4}}\right)\left(-5 r^{3}\left(m_{0}^{2}\left(R^{2}-r^{2}\right)+m_{0}\left(r^{6}+3 r^{2} R^{4}-4 R^{6}\right)\right.\right. \\
& \left.\left.+3 R^{4}\left(R^{6}-r^{6}\right)\right) F_{1}^{(2,0)}\left(\frac{r}{R}, \frac{m_{0}}{R^{4}}\right)+26 m_{0} R^{9}-22 R^{13}\right)
\end{aligned}
$$




$$
\begin{aligned}
& \left.+16 m_{0} R^{6}\left(m_{0}-R^{4}\right) F_{1}^{(1,1)}\left(\frac{r}{R}, \frac{m_{0}}{R^{4}}\right)\right)+96 m_{0} R^{7}\left(m_{0}-R^{4}\right) F_{1}^{(0,1)}\left(\frac{r}{R}, \frac{m_{0}}{R^{4}}\right) \\
& \left.\left.+288 r R^{6}\left(m_{0}-3 R^{4}\right)\left(m_{0}-R^{4}\right) F_{1}\left(\frac{r}{R}, \frac{m_{0}}{R^{4}}\right)^{2}\right)\right) \\
s_{6}^{(C)}= & \frac{2 \sqrt{3} \kappa\left(m_{0}-r^{4}\right)\left(R^{4}-m_{0}\right)\left(5 R F_{1}^{(1,0)}\left(\frac{r}{R}, \frac{m_{0}}{R^{4}}\right)+r F_{1}^{(2,0)}\left(\frac{r}{R}, \frac{m_{0}}{R^{4}}\right)\right)}{m_{0} R^{7}} .
\end{aligned}
$$

The source term $S_{h}$ is given by

$$
S_{h}=\sum_{i=1}^{6} s_{i}^{(h)} W_{s}^{i},
$$

where the functions $s_{i}^{(h)}$, s are given by

$$
\begin{aligned}
s_{1}^{(h)}= & \frac{1}{3 R(r+R)^{2}\left(-m_{0}+r^{4}+r^{2} R^{2}+R^{4}\right)^{2}}\left(2 r \left(2 \left(m_{0}\left(4 r^{3}+8 r^{2} R+6 r R^{2}+3 R^{3}\right)\right.\right.\right. \\
& \left.\left.\left.-3 R^{3}\left(r^{2}+r R+R^{2}\right)^{2}\right) F_{2}\left(\frac{r}{R}, \frac{m_{0}}{R^{4}}\right)+r^{2} R\left(r^{2}+r R+R^{2}\right)^{2}\right)\right), \\
s_{2}^{(h)}= & \frac{2}{3 r^{7}}\left(r^{4}-\frac{36 R^{4} \kappa^{2}\left(m_{0}-R^{4}\right)^{2}}{m_{0}^{2}}\right), \\
s_{3}^{(h)}= & 0, \\
s_{4}^{(h)}= & 0, \\
s_{5}^{(h)}= & \frac{r^{7}\left(R^{4}-m_{0}\right)}{R^{16}}\left(5 R F_{1}^{(1,0)}\left(\frac{r}{R}, \frac{m_{0}}{R^{4}}\right)+r F_{1}^{(2,0)}\left(\frac{r}{R}, \frac{m_{0}}{R^{4}}\right)\right)^{2} \\
s_{6}^{(h)}= & \frac{4 \sqrt{3} \kappa\left(R^{4}-m_{0}\right)}{m_{0} R^{7}}\left(5 R F_{1}^{(1,0)}\left(\frac{r}{R}, \frac{m_{0}}{R^{4}}\right)+r F_{1}^{(2,0)}\left(\frac{r}{R}, \frac{m_{0}}{R^{4}}\right)\right) .
\end{aligned}
$$

Finally the source term $S_{M}(r)$ is given by

$$
S_{M}(r)=\sum_{i=1}^{6} s_{i}^{(M)} W_{s}^{i},
$$


with the functions $s_{i}^{(M)}$ being given by

$$
\begin{aligned}
& s_{1}^{(M)}=\frac{4 r \sqrt{R^{2}\left(m_{0}-R^{4}\right)}\left(r^{2}+r R+R^{2}\right) F_{2}\left(\frac{r}{R}, \frac{m_{0}}{R^{4}}\right)}{R(r+R)\left(-m_{0}+r^{4}+r^{2} R^{2}+R^{4}\right)} \\
& s_{2}^{(M)}=-\frac{2 \sqrt{R^{2}\left(m_{0}-R^{4}\right)}\left(m_{0}^{2} r^{4}+12 R^{2} \kappa^{2}\left(m_{0}-R^{4}\right)\left(2 m_{0} r^{2}+3 m_{0} R^{2}-3 R^{6}\right)\right)}{m_{0}^{2} r^{7}} \\
& s_{3}^{(M)}=0 \\
& s_{4}^{(M)}=-\frac{r^{5} \sqrt{R^{2}\left(m_{0}-R^{4}\right)}\left(5 R F_{1}^{(1,0)}\left(\frac{r}{R}, \frac{m_{0}}{R^{4}}\right)+r F_{1}^{(2,0)}\left(\frac{r}{R}, \frac{m_{0}}{R^{4}}\right)\right)}{R^{9}} \\
& s_{5}^{(M)}=\frac{r^{5}\left(R^{2}\left(m_{0}-R^{4}\right)\right)^{3 / 2}}{R^{17}\left(3 R^{4}-m_{0}\right)}\left(r^{2}\left(-\left(6 r\left(m_{0}-3 R^{4}\right) F_{1}\left(\frac{r}{R}, \frac{m_{0}}{R^{4}}\right)+R^{5}\right)\right) F_{1}^{(3,0)}\left(\frac{r}{R}, \frac{m_{0}}{R^{4}}\right)\right. \\
& -2\left(15 r^{2} R\left(m_{0}-3 R^{4}\right) F_{1}^{(1,0)}\left(\frac{r}{R}, \frac{m_{0}}{R^{4}}\right)^{2}+\left(3 r ( m _ { 0 } - 3 R ^ { 4 } ) \left(r^{2} F_{1}^{(2,0)}\left(\frac{r}{R}, \frac{m_{0}}{R^{4}}\right)\right.\right.\right. \\
& \left.\left.+35 R^{2} F_{1}\left(\frac{r}{R}, \frac{m_{0}}{R^{4}}\right)\right)+20 R^{7}\right) F_{1}^{(1,0)}\left(\frac{r}{R}, \frac{m_{0}}{R^{4}}\right)+R\left(2 m_{0} r R F_{1}^{(2,1)}\left(\frac{r}{R}, \frac{m_{0}}{R^{4}}\right)\right. \\
& +r\left(39 r\left(m_{0}-3 R^{4}\right) F_{1}\left(\frac{r}{R}, \frac{m_{0}}{R^{4}}\right)+7 R^{5}\right) F_{1}^{(2,0)}\left(\frac{r}{R}, \frac{m_{0}}{R^{4}}\right) \\
& \left.\left.\left.+10 m_{0} R^{2} F_{1}^{(1,1)}\left(\frac{r}{R}, \frac{m_{0}}{R^{4}}\right)\right)\right)\right) \\
& s_{6}^{(M)}=\frac{\sqrt{3} \kappa\left(R^{4}-m_{0}\right)}{m_{0} r^{2} R^{7} \sqrt{R^{2}\left(m_{0}-R^{4}\right)}}\left(-m_{0} r^{4} R F_{1}^{(3,0)}\left(\frac{r}{R}, \frac{m_{0}}{R^{4}}\right)+r^{4} R^{5} F_{1}^{(3,0)}\left(\frac{r}{R}, \frac{m_{0}}{R^{4}}\right)\right. \\
& +r^{2} R\left(20 m_{0} r^{2}-17 m_{0} R^{2}+17 R^{6}\right) F_{1}^{(1,0)}\left(\frac{r}{R}, \frac{m_{0}}{R^{4}}\right) \\
& \left.+r^{3}\left(4 m_{0} r^{2}-7 m_{0} R^{2}+7 R^{6}\right) F_{1}^{(2,0)}\left(\frac{r}{R}, \frac{m_{0}}{R^{4}}\right)+4 R^{9}\right)
\end{aligned}
$$

\section{Source terms in vector sector: second order}

The source term in the vector sector at second order $S_{E}^{\mathrm{vec}}(r)$ in (4.19) is given by

$$
\left(S_{E}^{\mathrm{vec}}\right)_{i}(r)=\sum_{l=1}^{5} r_{l}^{(E)}\left(W_{v}\right)_{i}^{l}
$$


where the Weyl covariant quantities $W_{v}^{i}$ s are given in appendix A and the functions $s_{i}^{(E)}$ are given by

$$
\begin{aligned}
r_{1}^{(E)}= & \frac{r^{2}+r R+R^{2}}{3(r+R)\left(-m_{0}+r^{4}+r^{2} R^{2}+R^{4}\right)}, \\
r_{2}^{(E)}= & \frac{1}{3 r^{3}}, \\
r_{3}^{(E)}= & \frac{\kappa\left(R^{2}\left(m_{0}-R^{4}\right)\right)^{3 / 2}\left(m_{0}(r+2 R)+3 r\left(r^{2}+r R+R^{2}\right)^{2}\right)}{\sqrt{3} m_{0} r^{3}(r+R)^{2}\left(-m_{0}+r^{4}+r^{2} R^{2}+R^{4}\right)^{2}}, \\
r_{4}^{(E)}= & \frac{\left(m_{0}-R^{4}\right)}{3 R^{6}(r+R)^{2}\left(-m_{0}+r^{4}+r^{2} R^{2}+R^{4}\right)^{2}}\left(-6 r^{2}(r+R)\left(r^{2}+r R+R^{2}\right)\left(-m_{0}\right.\right. \\
& \left.+r^{4}+r^{2} R^{2}+R^{4}\right) F_{1}^{(1,0)}\left(\frac{r}{R}, \frac{m_{0}}{R^{4}}\right)-6 r R\left(3\left(r^{2}+r R+R^{2}\right)^{2}\left(r^{3}+2 R^{3}\right)\right. \\
& \left.-m_{0}\left(7 r^{3}+14 r^{2} R+12 r R^{2}+6 R^{3}\right)\right) F_{1}\left(\frac{r}{R}, \frac{m_{0}}{R^{4}}\right) \\
& \left.-\frac{R^{8}\left(m_{0}(2 r+R)+3 R\left(r^{2}+r R+R^{2}\right)^{2}\right)}{m_{0}-3 R^{4}}\right), \\
r_{5}^{(E)}= & \frac{\left(R^{4}-m_{0}\right)\left(r\left(9 R F_{1}^{(1,0)}\left(\frac{r}{R}, \frac{m_{0}}{R^{4}}\right)+r F_{1}^{(2,0)}\left(\frac{r}{R}, \frac{m_{0}}{R^{4}}\right)\right)+6 R^{2} F_{1}\left(\frac{r}{R}, \frac{m_{0}}{R^{4}}\right)\right)}{r^{2} R^{7}} .
\end{aligned}
$$

The other source term in the vector sector at second order $S_{M}^{\mathrm{vec}}(r)$ in (4.20) is given by

$$
\left(S_{M}^{\mathrm{vec}}\right)_{i}(r)=\sum_{l=1}^{5} r_{l}^{(M)}\left(W_{v}\right)_{i}^{l},
$$

where the coefficient functions $r_{i}^{(M)}$ are given by

$$
\begin{aligned}
r_{1}^{(M)}= & 0, \\
r_{2}^{(M)}= & \frac{2 \sqrt{3} \sqrt{R^{2}\left(m_{0}-R^{4}\right)}\left(m_{0} r^{2}+24 R^{2} \kappa^{2}\left(R^{4}-m_{0}\right)\right)}{m_{0} r^{5}}, \\
r_{3}^{(M)}= & \frac{6 R \kappa\left(m_{0}-R^{4}\right)}{m_{0} r^{5}(r+R)\left(-m_{0}+r^{4}+r^{2} R^{2}+R^{4}\right)}\left(r ^ { 2 } R \left(r\left(r^{2}+r R+R^{2}\right)\left(3 r^{3}+R^{3}\right)\right.\right. \\
& \left.\left.-m_{0}\left(3 r^{2}+3 r R+2 R^{2}\right)\right)-8 m_{0}(r+R)\left(-m_{0}+r^{4}+r^{2} R^{2}+R^{4}\right) F_{2}\left(\frac{r}{R}, \frac{m_{0}}{R^{4}}\right)\right), \\
r_{4}^{(M)}= & -\frac{2 \sqrt{3} \sqrt{R^{2}\left(m_{0}-R^{4}\right)}}{R^{6}(r+R)\left(-m_{0}+r^{4}+r^{2} R^{2}+R^{4}\right)}\left(r ^ { 2 } ( r + R ) \left(-m_{0}+r^{4}\right.\right. \\
& \left.+r^{2} R^{2}+R^{4}\right)\left(5 R F_{1}^{(1,0)}\left(\frac{r}{R}, \frac{m_{0}}{R^{4}}\right)+r F_{1}^{(2,0)}\left(\frac{r}{R}, \frac{m_{0}}{R^{4}}\right)\right) \\
& \left.+12 r R\left(m_{0}-R^{4}\right)\left(r^{2}+r R+R^{2}\right) F_{1}\left(\frac{r}{R}, \frac{m_{0}}{R^{4}}\right)+R^{6}\left(r^{2}+r R+R^{2}\right)\right), \\
r_{5}^{(M)}= & \frac{2 \sqrt{3} \sqrt{R^{2}\left(m_{0}-R^{4}\right)}}{m_{0} r^{5} R^{6}}\left(6 m_{0} r^{3}\left(m_{0}-R^{4}\right)\left(R F_{1}\left(\frac{r}{R}, \frac{m_{0}}{R^{4}}\right)-r F_{1}^{(1,0)}\left(\frac{r}{R}, \frac{m_{0}}{R^{4}}\right)\right)\right. \\
& \left.+m_{0} r^{2} R^{6}+24 R^{8} \kappa^{2}\left(R^{4}-m_{0}\right)\right) .
\end{aligned}
$$




\section{Source terms in tensor sector: second order}

In this appendix we provide the source of the dynamical equation (4.32). We report the result in terms of the parameters $M$ and $R$ and the variable $\rho$ defined in (2.5). The source $\mathbf{T}_{i j}(\rho)$ in (4.32) is given by

$$
\mathbf{T}_{i j}(r)=\sum_{l=1}^{9} \tau_{l}(r) W T_{i j}^{(l)},
$$

where the weyl-covariant terms $W T_{i j}^{(l)}$ are defined in appendix A in equation (A.10). The coefficient of the weyl-covariant terms in the above source is given by

$$
\begin{aligned}
\tau_{1}(r)= & \frac{3 r F_{2}\left(\frac{r}{R}, \frac{m_{0}}{R^{4}}\right)}{R}+\frac{m_{0}(r+R)-\left(r^{2}+r R+R^{2}\right)\left(3 r^{3}+R^{3}\right)}{(r+R)\left(-m_{0}+r^{4}+r^{2} R^{2}+R^{4}\right)}, \\
\tau_{2}(r)= & -\frac{1}{2 R}\left(3 r F_{2}\left(\frac{r}{R}, \frac{m_{0}}{R^{4}}\right)-\frac{2 r^{3} R\left(r^{2}+r R+R^{2}\right)}{(r+R)\left(-m_{0}+r^{4}+r^{2} R^{2}+R^{4}\right)}+R\right), \\
\tau_{3}(r)= & \frac{6 r F_{2}\left(\frac{r}{R}, \frac{m_{0}}{R^{4}}\right)}{R}+\frac{2\left(m_{0}(r+R)-2 r^{3}\left(r^{2}+r R+R^{2}\right)\right)}{(r+R)\left(-m_{0}+r^{4}+r^{2} R^{2}+R^{4}\right)}, \\
\tau_{4}(r)= & \frac{18 R^{4} \kappa^{2}\left(m_{0}-R^{4}\right)^{2}\left(-m_{0} r^{2}+4 m_{0} R^{2}+r^{6}-4 R^{6}\right)}{m_{0}^{2} r^{10}}-\frac{m_{0} r^{2}+2 m_{0} R^{2}+r^{6}-2 R^{6}}{2 r^{6}}, \\
\tau_{5}(r)= & \frac{1}{R^{6}}\left(6 r\left(R^{4}-m_{0}\right)\left(r F_{1}^{(1,0)}\left(\frac{r}{R}, \frac{m_{0}}{R^{4}}\right)+3 R F_{1}\left(\frac{r}{R}, \frac{m_{0}}{R^{4}}\right)\right)\right), \\
\tau_{6}(r)= & \frac{3 r\left(m_{0}-R^{4}\right)}{2 R^{16}\left(m_{0}-3 R^{4}\right)}\left(r \left(R ^ { 2 } F _ { 1 } ^ { ( 1 , 0 ) } ( \frac { r } { R } , \frac { m _ { 0 } } { R ^ { 4 } } ) \left(r ^ { 2 } ( m _ { 0 } - 3 R ^ { 4 } ) \left(-25 m_{0} r^{2}\right.\right.\right.\right. \\
& \left.\left.+37 m_{0} R^{2}+25 r^{6}-37 R^{6}\right) F_{1}^{(1,0)}\left(\frac{r}{R}, \frac{m_{0}}{R^{4}}\right)+30 m_{0} R^{8}-2 R^{12}\right) \\
& -r^{4}\left(3 R^{4}-m_{0}\right)(r-R)(r+R)\left(-m_{0}+r^{4}+r^{2} R^{2}+R^{4}\right) F_{1}^{(2,0)}\left(\frac{r}{R}, \frac{m_{0}}{R^{4}}\right)^{2} \\
& +2 r R\left(5 r ^ { 2 } ( 3 R ^ { 4 } - m _ { 0 } ) ( R - r ) ( r + R ) \left(-m_{0}+r^{4}\right.\right. \\
& \left.\left.+r^{2} R^{2}+R^{4}\right) F_{1}^{(1,0)}\left(\frac{r}{R}, \frac{m_{0}}{R^{4}}\right)+R^{8}\left(m_{0}+R^{4}\right)\right) F_{1}^{(2,0)}\left(\frac{r}{R}, \frac{m_{0}}{R^{4}}\right) \\
& \left.+16 m_{0} R^{6}\left(m_{0}-R^{4}\right) F_{1}^{(1,1)}\left(\frac{r}{R}, \frac{m_{0}}{R^{4}}\right)\right)+48 m_{0} R^{7}\left(m_{0}-R^{4}\right) F_{1}^{(0,1)}\left(\frac{r}{R}, \frac{m_{0}}{R^{4}}\right) \\
\tau_{8}(r)= & \frac{3 \sqrt{3} \kappa\left(R^{2}\left(m_{0}-R^{4}\right)\right)^{3 / 2}}{m_{0} r^{5} R^{9}}\left(2 r ^ { 2 } \left(R\left(-m_{0}\left(5 r^{2}+R^{2}\right)+5 r^{6}+R^{6}\right) F_{1}^{(1,0)}\left(\frac{r}{R}, \frac{m_{0}}{R^{4}}\right)\right.\right. \\
& \left.+24 R^{11}\left(3 m_{0}-2 R^{4}\right) F_{1}\left(\frac{r}{R}, \frac{m_{0}}{R^{4}}\right)\right) \\
\tau_{7}(r)= & \left.\left.\left.\frac{3 \sqrt{3} \kappa\left(R^{2}\left(m_{0}-R^{4}\right)\right)^{3 / 2}}{2}, \frac{m_{0}}{R^{4}}\right)\right)+R^{9}\right), \\
& \left.+R^{6}\right) F_{1}^{(2,0)}\left(\frac{r}{m^{5}}\right) \\
& \\
& \\
&
\end{aligned}
$$




\section{E Comparison with Erdmenger et al. [61]}

Firstly we shall present a dictionary of relations between the quantities defined in [61] and those in this paper. To avoid confusion we shall use a subscript ' $E$ ' to denote the quantities in [61].

The charge and mass of the black brane in the two papers are related by

$$
\begin{aligned}
(Q)_{E} & =-q \\
(b)_{E}^{4} & =\frac{1}{m} .
\end{aligned}
$$

Also the gauge field in [61] is twice the gauge field in our paper

$$
\left(A_{\mu}\right)_{E}=2 A_{\mu} .
$$

We list the relation between several other quantities in the two papers

$$
\begin{aligned}
\left(r_{+}\right)_{E} & =R \\
\left(r_{-}\right)_{E} & =R \sqrt{\left(Q^{2}+\frac{1}{4}\right)^{\frac{1}{2}}-\frac{1}{2}} \\
\mu_{E} & =-\frac{\sqrt{3} q}{R^{2}}=-2 \mu \\
T_{E} & =\frac{R}{2 \pi}(3-M)=\frac{R}{2 \pi}\left(2-Q^{2}\right) \\
N_{E}^{2} & =\frac{\pi}{2 G_{5}} \\
\left(\sigma_{\mu \nu}\right)_{E} & =2 \sigma_{\mu \nu} \\
\left(l_{\mu}\right)_{E} & =-l_{\mu} .
\end{aligned}
$$

Finally the various functions that go into the first order metric and the gauge field are related by

$$
\begin{aligned}
(F(r))_{E} & =\frac{1}{R} F_{2}(\rho, M) \\
\left(j^{\kappa}(r)\right)_{E} & =\frac{\sqrt{3} Q\left(2-Q^{2}\right)^{3}}{2 \pi R\left(2+3 Q^{2}+Q^{4}\right)} F_{1}(\rho, M) \\
\left(a^{\kappa}(r)\right)_{E} & =-\frac{\rho^{5}\left(2-Q^{2}\right)^{3}}{4 \pi\left(2+3 Q^{2}+Q^{4}\right)} F_{1}^{(1,0)}(\rho, M) .
\end{aligned}
$$

These statements are true only up to zeroth order in the expansion of $\mathrm{R}$ in terms of the boundary derivatives. Further for the tensor sector matching we have to use the following relations

$$
\begin{aligned}
\mathcal{D}_{i}\left(\frac{\mu}{T}\right) & =\frac{2 \pi \sqrt{3}\left(2+3 Q^{2}+Q^{4}\right)}{R^{3}\left(2-Q^{2}\right)^{3}} \mathcal{D}_{i} q \\
\mathcal{D}_{i} \mathcal{D}_{j}\left(\frac{\mu}{T}\right) & =\frac{2 \pi \sqrt{3}\left(2+3 Q^{2}+Q^{4}\right)}{R^{3}\left(2-Q^{2}\right)^{3}} \mathcal{D}_{i} \mathcal{D}_{j} q+\frac{2 \pi \sqrt{3} Q\left(1+Q^{2}\right)\left(60+40 Q^{2}+Q^{4}\right)}{R^{6}\left(2-Q^{2}\right)^{5}} \mathcal{D}_{i} q \mathcal{D}_{j} q
\end{aligned}
$$


where,

$$
\mu=\frac{\sqrt{3} q}{2 R^{2}} ; \quad T=\frac{R}{2 \pi}\left(2-Q^{2}\right),
$$

are respectively the chemical potential and the temperature in our notation.

Using this dictionary our stress tensor and charge current mathches perfectly with [61].

Open Access. This article is distributed under the terms of the Creative Commons Attribution Noncommercial License which permits any noncommercial use, distribution, and reproduction in any medium, provided the original author(s) and source are credited.

\section{References}

[1] S. Bhattacharyya, V.E. Hubeny, S. Minwalla and M. Rangamani, Nonlinear Fluid Dynamics from Gravity, JHEP 02 (2008) 045 [arXiv:0712.2456] [SPIRES].

[2] S. Bhattacharyya et al., Local Fluid Dynamical Entropy from Gravity, JHEP 06 (2008) 055 [arXiv: 0803.2526] [SPIRES].

[3] S. Bhattacharyya et al., Forced Fluid Dynamics from Gravity, JHEP 02 (2009) 018 [arXiv:0806.0006] [SPIRES].

[4] M. Haack and A. Yarom, Nonlinear viscous hydrodynamics in various dimensions using AdS/CFT, JHEP 10 (2008) 063 [arXiv:0806.4602] [SPIRES].

[5] M. Van Raamsdonk, Black Hole Dynamics From Atmospheric Science, JHEP 05 (2008) 106 [arXiv: 0802.3224] [SPIRES].

[6] R. Loganayagam, Entropy Current in Conformal Hydrodynamics, JHEP 05 (2008) 087 [arXiv:0801.3701] [SPIRES].

[7] M.P. Heller, P. Surowka, R. Loganayagam, M. Spalinski and S.E. Vazquez, On a consistent AdS/CFT description of boost-invariant plasma, arXiv:0805.3774 [SPIRES].

[8] S. Dutta, Higher Derivative Corrections to Locally Black Brane Metrics, JHEP 05 (2008) 082 [arXiv:0804.2453] [SPIRES].

[9] G. Policastro, D.T. Son and A.O. Starinets, The shear viscosity of strongly coupled $N=4$ supersymmetric Yang-Mills plasma, Phys. Rev. Lett. 87 (2001) 081601 [hep-th/0104066] [SPIRES].

[10] R.A. Janik and R.B. Peschanski, Asymptotic perfect fluid dynamics as a consequence of AdS/CFT, Phys. Rev. D 73 (2006) 045013 [hep-th/0512162] [SPIRES].

[11] R.A. Janik and R.B. Peschanski, Gauge/gravity duality and thermalization of a boost-invariant perfect fluid, Phys. Rev. D 74 (2006) 046007 [hep-th/0606149] [SPIRES].

[12] S. Nakamura and S.-J. Sin, A holographic dual of hydrodynamics, JHEP 09 (2006) 020 [hep-th/0607123] [SPIRES].

[13] S.-J. Sin, S. Nakamura and S.P. Kim, Elliptic Flow, Kasner Universe and Holographic Dual of RHIC Fireball, JHEP 12 (2006) 075 [hep-th/0610113] [SPIRES].

[14] R.A. Janik, Viscous plasma evolution from gravity using AdS/CFT, Phys. Rev. Lett. 98 (2007) 022302 [hep-th/0610144] [SPIRES]. 
[15] J.J. Friess, S.S. Gubser, G. Michalogiorgakis and S.S. Pufu, Expanding plasmas and quasinormal modes of anti-de Sitter black holes, JHEP 04 (2007) 080 [hep-th/0611005] [SPIRES].

[16] K. Kajantie and T. Tahkokallio, Spherically expanding matter in AdS/CFT, Phys. Rev. D 75 (2007) 066003 [hep-th/0612226] [SPIRES].

[17] P. Benincasa, A. Buchel, M.P. Heller and R.A. Janik, On the supergravity description of boost invariant conformal plasma at strong coupling, Phys. Rev. D 77 (2008) 046006 [arXiv:0712.2025] [SPIRES].

[18] C.P. Herzog, The hydrodynamics of M-theory, JHEP 12 (2002) 026 [hep-th/0210126] [SPIRES].

[19] G. Policastro, D.T. Son and A.O. Starinets, From AdS/CFT correspondence to hydrodynamics. II: Sound waves, JHEP 12 (2002) 054 [hep-th/0210220] [SPIRES].

[20] G. Policastro, D.T. Son and A.O. Starinets, From AdS/CFT correspondence to hydrodynamics, JHEP 09 (2002) 043 [hep-th/0205052] [SPIRES].

[21] D.T. Son and A.O. Starinets, Minkowski-space correlators in AdS/CFT correspondence: Recipe and applications, JHEP 09 (2002) 042 [hep-th/0205051] [SPIRES].

[22] C.P. Herzog and D.T. Son, Schwinger-Keldysh propagators from AdS/CFT correspondence, JHEP 03 (2003) 046 [hep-th/0212072] [SPIRES].

[23] C.P. Herzog, The sound of M-theory, Phys. Rev. D 68 (2003) 024013 [hep-th/0302086] [SPIRES].

[24] P. Kovtun, D.T. Son and A.O. Starinets, Holography and hydrodynamics: Diffusion on stretched horizons, JHEP 10 (2003) 064 [hep-th/0309213] [SPIRES].

[25] A. Buchel and J.T. Liu, Universality of the shear viscosity in supergravity, Phys. Rev. Lett. 93 (2004) 090602 [hep-th/0311175] [SPIRES].

[26] A. Buchel, J.T. Liu and A.O. Starinets, Coupling constant dependence of the shear viscosity in $N=4$ supersymmetric Yang-Mills theory, Nucl. Phys. B 707 (2005) 56 [hep-th/0406264] [SPIRES].

[27] A. Buchel, On universality of stress-energy tensor correlation functions in supergravity, Phys. Lett. B 609 (2005) 392 [hep-th/0408095] [SPIRES].

[28] P. Kovtun, D.T. Son and A.O. Starinets, Viscosity in strongly interacting quantum field theories from black hole physics, Phys. Rev. Lett. 94 (2005) 111601 [hep-th/0405231] [SPIRES].

[29] P.K. Kovtun and A.O. Starinets, Quasinormal modes and holography, Phys. Rev. D 72 (2005) 086009 [hep-th/0506184] [SPIRES].

[30] P. Benincasa, A. Buchel and A.O. Starinets, Sound waves in strongly coupled non-conformal gauge theory plasma, Nucl. Phys. B 733 (2006) 160 [hep-th/0507026] [SPIRES].

[31] K. Maeda, M. Natsuume and T. Okamura, Viscosity of gauge theory plasma with a chemical potential from AdS/CFT, Phys. Rev. D 73 (2006) 066013 [hep-th/0602010] [SPIRES].

[32] J. Mas, Shear viscosity from R-charged AdS black holes, JHEP 03 (2006) 016 [hep-th/0601144] [SPIRES]. 
[33] O. Saremi, The viscosity bound conjecture and hydrodynamics of M2-brane theory at finite chemical potential, JHEP 10 (2006) 083 [hep-th/0601159] [SPIRES].

[34] D.T. Son and A.O. Starinets, Hydrodynamics of R-charged black holes, JHEP 03 (2006) 052 [hep-th/0601157] [SPIRES].

[35] P. Benincasa, A. Buchel and R. Naryshkin, The shear viscosity of gauge theory plasma with chemical potentials, Phys. Lett. B 645 (2007) 309 [hep-th/0610145] [SPIRES].

[36] K.B. Fadafan, Charge effect and finite 't Hooft coupling correction on drag force and Jet Quenching Parameter, Eur. Phys. J. C 68 (2010) 505 [arXiv:0809.1336] [SPIRES].

[37] D.T. Son and A.O. Starinets, Viscosity, Black Holes and Quantum Field Theory, Ann. Rev. Nucl. Part. Sci. 57 (2007) 95 [arXiv:0704.0240] [SPIRES].

[38] E. Shuryak, Why does the quark gluon plasma at RHIC behave as a nearly ideal fluid?, Prog. Part. Nucl. Phys. 53 (2004) 273 [hep-ph/0312227] [SPIRES].

[39] E.V. Shuryak, What RHIC experiments and theory tell us about properties of quark-gluon plasma?, Nucl. Phys. A 750 (2005) 64 [hep-ph/0405066] [SPIRES].

[40] E.V. Shuryak, Strongly coupled quark-gluon plasma: The status report, hep-ph/0608177 [SPIRES].

[41] J.-L. Gervais and B. Sakita, Quantized relativistic string as a strong coupling limit of the Higgs model, Nucl. Phys. B 91 (1975) 301 [SPIRES].

[42] R. Baier, P. Romatschke, D.T. Son, A.O. Starinets and M.A. Stephanov, Relativistic viscous hydrodynamics, conformal invariance and holography, JHEP 04 (2008) 100 [arXiv:0712.2451] [SPIRES].

[43] M. Henningson and K. Skenderis, The holographic Weyl anomaly, JHEP 07 (1998) 023 [hep-th/9806087] [SPIRES].

[44] V. Balasubramanian and P. Kraus, A stress tensor for anti-de Sitter gravity, Commun. Math. Phys. 208 (1999) 413 [hep-th/9902121] [SPIRES].

[45] S. Bhattacharyya, S. Lahiri, R. Loganayagam and S. Minwalla, Large rotating AdS black holes from fluid mechanics, JHEP 09 (2008) 054 [arXiv:0708.1770] [SPIRES].

[46] A. Buchel, R.C. Myers, M.F. Paulos and A. Sinha, Universal holographic hydrodynamics at finite coupling, Phys. Lett. B 669 (2008) 364 [arXiv:0808.1837] [SPIRES].

[47] A. Buchel and M. Paulos, Second order hydrodynamics of a CFT plasma from boost invariant expansion, Nucl. Phys. B $\mathbf{8 1 0}$ (2009) 40 [arXiv:0808.1601] [SPIRES].

[48] M. Cvetič and S.S. Gubser, Thermodynamic Stability and Phases of General Spinning Branes, JHEP 07 (1999) 010 [hep-th/9903132] [SPIRES].

[49] A. Chamblin, R. Emparan, C.V. Johnson and R.C. Myers, Charged AdS black holes and catastrophic holography, Phys. Rev. D 60 (1999) 064018 [hep-th/9902170] [SPIRES].

[50] A. Chamblin, R. Emparan, C.V. Johnson and R.C. Myers, Holography, thermodynamics and fluctuations of charged AdS black holes, Phys. Rev. D 60 (1999) 104026 [hep-th/9904197] [SPIRES].

[51] D. Astefanesei, N. Banerjee and S. Dutta, (Un)attractor black holes in higher derivative AdS gravity, JHEP 11 (2008) 070 [arXiv:0806.1334] [SPIRES]. 
[52] D. Astefanesei, H. Nastase, H. Yavartanoo and S. Yun, Moduli flow and non-supersymmetric AdS attractors, JHEP 04 (2008) 074 [arXiv: 0711.0036] [SPIRES].

[53] R.-G. Cai and A. Wang, Thermodynamics and stability of hyperbolic charged black holes, Phys. Rev. D 70 (2004) 064013 [hep-th/0406057] [SPIRES].

[54] R.-G. Cai and K.-S. Soh, Critical behavior in the rotating D-branes, Mod. Phys. Lett. A 14 (1999) 1895 [hep-th/9812121] [SPIRES].

[55] M. Cvetič et al., Embedding AdS black holes in ten and eleven dimensions, Nucl. Phys. B 558 (1999) 96 [hep-th/9903214] [SPIRES].

[56] J.T. Liu and W.A. Sabra, Mass in anti-de Sitter spaces, Phys. Rev. D 72 (2005) 064021 [hep-th/0405171] [SPIRES].

[57] M. Cvetič and S.S. Gubser, Phases of R-charged black holes, spinning branes and strongly coupled gauge theories, JHEP 04 (1999) 024 [hep-th/9902195] [SPIRES].

[58] S.S. Gubser, Thermodynamics of spinning D3-branes, Nucl. Phys. B 551 (1999) 667 [hep-th/9810225] [SPIRES].

[59] N. Banerjee and S. Dutta, Phase Transition of Electrically Charged Ricci-flat Black Holes, JHEP 07 (2007) 047 [arXiv: 0705.2682] [SPIRES].

[60] D. Marolf, Chern-Simons terms and the three notions of charge, hep-th/0006117 [SPIRES].

[61] J. Erdmenger, M. Haack, M. Kaminski and A. Yarom, Fluid dynamics of R-charged black holes, JHEP 01 (2009) 055 [arXiv:0809.2488] [SPIRES]. 